\title{
CONTROL-ORIENTED PLANAR MOTION MODELING OF UNMANNED SURFACE VEHICLES
}

C. Sonnenburg, A. Gadre, D. Horner, S. Krageland, A. Marcus, D. J. Stilwell, and C. A. Woolsey

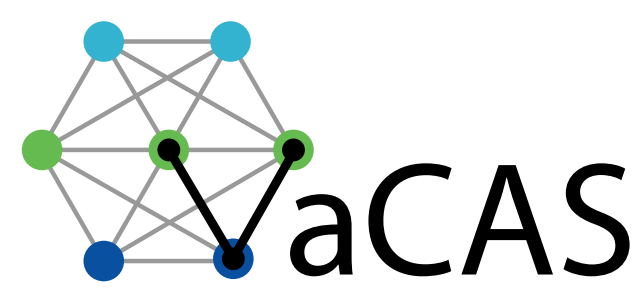

Virginia Center for Autonomous Systems Virginia Polytechnic Institute \& State University

Blacksburg, VA 24060

www . unmanned.vt. edu

September 16, 2010

Technical Report No. VaCAS-2010-01

Copyright (C) 2010 


\section{Summary}

This technical report describes a comparison of experimentally identified dynamic models for the planar motion of an unmanned surface vehicle (USV). The objective is to determine a model which is (1) sufficiently rich to enable effective model-based control design, (2) sufficiently simple to allow straight forward parameter identification, and (3) sufficiently general to apply to a variety of hullforms and actuator configurations. Starting from a three degree-of-freedom nonlinear model obtained from physical principles, we consider five simplified variants that include four linear models and two nonlinear models for low speed operation. The first linear model comes from linearizing the full planar boat dynamics about a straight constant speed. A first order steering model relates steering angle to turn rate. A second order steering model relates steering angle to turn rate and sideslip angle. A first order speed model relates throttle setting to forward speed. The two nonlinear models are derived from potential flow around a simple shape. Linear damping and quadratic damping are included in each nonlinear model respectively. To identify parameters for these models, data must be collected that show the dynamic and steady-state relationships between inputs and outputs. Using these data sets, simple models that satisfy the three given criteria are identified for three types of unmanned surface vehicle: a rigid hull inflatable boat with an

outboard engine, a rigid hull inflatable boat with a waterjet propulsion system, and a small pontoon boat with two electric thrusters. 


\section{Contents}

1 Introduction $\quad 1$

2 Dynamic Modeling $\quad 2$

2.1 Notation and Reference Frames . . . . . . . . . . . . . . . . . . . 2

2.2 Nonlinear Planar Model . . . . . . . . . . . . . . . . . . . 5

2.3 Linear Models . . . . . . . . . . . . . . . . . . . . . . . . . 7

2.4 Low-Speed Nonlinear Model . . . . . . . . . . . . . . . . . . 8

3 Model Identification $\quad 9$

3.1 Identifying Steady-State Input/Output Relationships . . . . . . . . . . . . 10

3.2 Enforcing Steady-State Input/Output Relationships When Identifying Dynamic Parameters . . . . . . . . . . . . . . . . . . . . 11

3.3 Initializing the Algorithm that Identifies Dynamic Parameters . . . . . . . 12

3.4 Identifying Dynamic Parameters . . . . . . . . . . . . . . . . . 13

4 Platforms $\quad 14$

4.1 Ribcraft USV . . . . . . . . . . . . . . . . . . . . . . 14

4.2 SeaFox USV . . . . . . . . . . . . . . . . . . . . . . 14

4.3 Pontoon USV . . . . . . . . . . . . . . . . . . . . . . . . 17

5 System Identification Results $\quad 18$

5.1 Steady-State Input/Output Parameters . . . . . . . . . . . . . . . 18

5.2 Actuator Dynamics . . . . . . . . . . . . . . . . . 23

5.3 Dynamics from Input/Output Data . . . . . . . . . . . . . . . 23

6 Conclusion $\quad 27$

$\begin{array}{lr}\text { Appendices } & 29\end{array}$

A Ribcraft USV Parameters and Time Histories $\quad 29$

A.1 Parameters . . . . . . . . . . . . . . . . . . . . . 29

A.2 Time Histories . . . . . . . . . . . . . . . . . . . . . . . . . . . . . . . 29 
B Seafox USV Parameters and Time Histories 40

B.1 Parameters . . . . . . . . . . . . . . . . . . 40

B.2 Time Histories . . . . . . . . . . . . . . . . . . . . . . . 40

C Pontoon USV Parameters and Time Histories 43

C.1 Parameters . . . . . . . . . . . . . . . . . . . . . 43

C.2 Time Histories . . . . . . . . . . . . . . . . . . . . . . . . . . . 43 


\section{List of Figures}

1 Schematic depiction of a USV . . . . . . . . . . . . . . . . . 4

2 Definitions of sideslip and course angles. . . . . . . . . . . . . 5

3 Closed-loop control scheme. Additional signals used in model identification are shown as outputs. . . . . . . . . . . . . . . . 13

4 Ribcraft USV on Peak Creek . . . . . . . . . . . . . . . . . . . 15

5 SeaFox operating on the Sacramento River . . . . . . . . . . . . . 16

6 Pontoon USV operating in a pond on the VT campus . . . . . . . . . . 18

$7 \quad$ Ribcraft USV steady-state data. . . . . . . . . . . . . . . . . . . . 20

8 Seafox USV steady-state data. . . . . . . . . . . . . . . . 21

9 Pontoon USV steady-state data. . . . . . . . . . . . . . . . . . . 22

10 Time histories for speed (actual and simulated) for the Ribcraft in straight-line motion. . . . . . . . . . . . . . . . . . . 24

11 Representative time histories of course angle (actual and simulated) for the Ribcraft USV in straight-line motion. . . . . . . . . . . . . . . . 25

12 Time histories of course angle (actual and simulated) for the Pontoon USV in straight-line motion. . . . . . . . . . . . . . . 25

13 Time histories of course angle (actual and simulated) for the Seafox USV in straight-line motion. . . . . . . . . . . . . . . . . 26

14 Time histories for course angle (actual and simulated) for the Ribcraft USV at $20 \%$ throttle. . . . . . . . . . . . . . . . . . 31

15 Time histories for course angle (actual and simulated) for the Ribcraft USV at $30 \%$ throttle. . . . . . . . . . . . . . . . . . 31

16 Time histories for course angle (actual and simulated) for the Ribcraft USV at $35 \%$ throttle. . . . . . . . . . . . . . . . . . . 32

17 Time histories for course angle (actual and simulated) for the Ribcraft USV at $40 \%$ throttle. . . . . . . . . . . . . . . . . . . 32

18 Time histories for course angle (actual and simulated) for the Ribcraft USV at $45 \%$ throttle. . . . . . . . . . . . . . . . . . . . . .

19 Time histories for course angle (actual and simulated) for the Ribcraft USV at $50 \%$ throttle. . . . . . . . . . . . . . . . . .

20 Time histories for course angle (actual and simulated) for the Ribcraft USV at $55 \%$ throttle. . . . . . . . . . . . . . . . . . 
21 Time histories for course angle (actual and simulated) for the Ribcraft USV at $60 \%$ throttle. . . . . . . . . . . . . . . . . . 34

22 Time histories for course angle (actual and simulated) for the Ribcraft USV at $65 \%$ throttle. . . . . . . . . . . . . . . . . . . .

23 Time histories for speed (actual and simulated) for the Ribcraft USV for straight line travel around $1 \mathrm{~m} / \mathrm{s} \ldots \ldots . \ldots . . \ldots 36$

24 Time histories for speed (actual and simulated) for the Ribcraft USV for straight line travel around $1.5 \mathrm{~m} / \mathrm{s} . \ldots \ldots \ldots$

25 Time histories for speed (actual and simulated) for the Ribcraft USV for

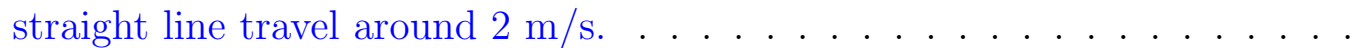

26 Time histories for speed (actual and simulated) for the Ribcraft USV for

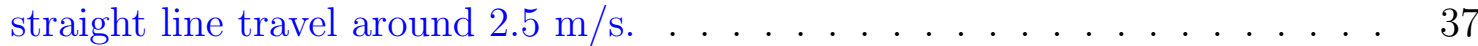

27 Time histories for speed (actual and simulated) for the Ribcraft USV for straight line travel around $3 \mathrm{~m} / \mathrm{s} . \ldots \ldots \ldots$. . . . . . . . . .

28 Time histories for speed (actual and simulated) for the Ribcraft USV for

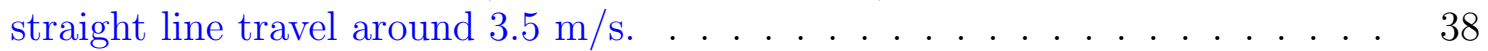

29 Time histories for speed (actual and simulated) for the Ribcraft USV for straight line travel around $4 \mathrm{~m} / \mathrm{s} . \ldots \ldots \ldots$

30 Time histories for course angle (actual and simulated) for the Seafox USV at $15 \%$ throttle. . . . . . . . . . . . . . . . . . . .

31 Time histories for course angle (actual and simulated) for the Seafox USV at 20\% throttle. . . . . . . . . . . . . . . . . 41

32 Time histories for course angle (actual and simulated) for the Seafox USV at 25\% throttle. . . . . . . . . . . . . . . . . . 42

33 Time histories for course angle (actual and simulated) for the Pontoon USV at $40 \%$ throttle. . . . . . . . . . . . . . . . . . . 44

34 Time histories for course angle (actual and simulated) for the Pontoon USV at $50 \%$ throttle. . . . . . . . . . . . . . . . . . . . . 


\section{List of Tables}

1 Steady-state values of $u, \beta$, and $r$ for the Ribcraft USV . . . . . . . . . . 20

2 Steady-state values of $u, \beta$, and $r$ for the Seafox USV . . . . . . . . . . . 21

3 Steady-state values of $u, \beta$, and $r$ for the Pontoon USV . . . . . . . . . . 21

$4 \quad$ Steady-state DC gains of $u, \beta, v$, and $r$ for the Ribcraft USV. . . . . . . . 22

$5 \quad$ Steady-state DC gains of $\beta, v$, and $r$ for the Seafox USV . . . . . . . . . 22

6 Steady-state DC gains of $\beta, v$, and $r$ for the Pontoon USV . . . . . . . . 23

7 Steering cost function values for the Ribcraft USV . . . . . . . . . . . . . 26

8 Speed cost function values for the Ribcraft USV . . . . . . . . . . . . . 26

9 Steering cost function values for the Pontoon USV . . . . . . . . . . . . . . 27

10 Steering cost function values for the Seafox USV . . . . . . . . . . . . . 27

11 Ribcraft USV nonlinear (??) parameter values (using linearly parameterized fit of steady-state gains). . . . . . . . . . . . . . . . . . . . 29

12 Ribcraft USV linear (21) parameter values (using linear fit of steady-state gains). . . . . . . . . . . . . . . . . . . . . . . . . 29

13 Ribcraft USV Nomoto-with-sideslip (25) parameter values (using linear fit of steady-state gains). . . . . . . . . . . . . . . . . . . . . . . 30

14 Ribcraft USV Nomoto (23) parameter values (using linear fit of steady-state gains). . . . . . . . . . . . . . . . . . .

15 Ribcraft USV speed (19) parameter values (using linear fit of steady-state gains). . . . . . . . . . . . . . . . . . 30

16 Seafox USV linear (21) parameter values (using linear fit of steady-state gains). 40

17 Seafox USV Nomoto-with-sideslip (25) parameter values (using linear fit of steady-state gains). . . . . . . . . . . . . . . . . . . . . . 40

18 Seafox USV Nomoto (23) parameter values (using linear fit of steady-state gains). . . . . . . . . . . . . . . . . . . . 40

19 Pontoon USV linear (21) parameter values (using linear fit of steady-state gains). . . . . . . . . . . . . . . . . . .

20 Pontoon USV Nomoto-with-sideslip (25) parameter values (using linear fit of steady-state gains). . . . . . . . . . . . . . . . . . . . . 43

21 Pontoon USV Nomoto (23) parameter values (using linear fit of steady-state gains). 


\section{Introduction}

Unmanned surface vehicles (USVs) fill an increasingly important niche in the pantheon of robotic vehicles. Equipped with appropriate sensors, USVs can collect information about the subsurface and above-surface environments and relay that information at a high data rate as it is collected. As part of a heterogeneous autonomous vehicle network, a USV can provide a mobile interface between ground, air, and space assets that communicate via radio frequency and submerged assets that communicate acoustically. Using combustion engines for propulsion and electrical power, and with large fuel and payload capacities, USVs offer exceptional endurance and operational capabilities. Using an attitude and heading reference system (AHRS) to complement GPS position measurements, a USV can maintain an accurate estimate of its own state and can also infer environmental characteristics, such as local flow speed and direction. Overviews of recent USV development activities can be found in [9, 2]. Platforms and applications related to defense and security are described in [8].

One application for USVs is rapid mapping of a riverine environment, where waterways may be narrow and non-uniform in depth and flow [1]. Such environments may be poorly known because of traffic and debris, shifting bathymetry, variable water levels, new construction, or other factors. A riverine USV, acting alone or in a team, can characterize such an environment, providing bathymetric maps to a human supervisor, for example. To operate in such an environment, however, the USV must be able to autonomously navigate in a dynamic, uncertain waterway. Moreover, to provide a rapid assessment, the perception and maneuvering algorithms must execute quickly and reliably. This paper focuses on USV dynamic modeling as it relates to agile autonomous maneuvering in the confines of a riverine waterway. The objective is to determine a model which is (1) sufficiently rich to enable effective motion planning and control, (2) sufficiently simple to allow straight forward parameter identification, and (3) sufficiently general to describe a variety of hullforms and actuator configurations. We consider a set of simple models whose parameters can be quickly and easily identified from standard motion data. Having selected an appropriate model, one may then use it to generate dynamically feasible trajectories for the USV to follow.

Virginia Tech and the Naval Postgraduate School (NPS) have developed and operate a number of autonomous marine vehicles, including several USVs. The platforms considered here represent three distinct control system implementations: a hydraulically actuated outboard engine, a hydraulically actuated jet-drive thruster, and differential electric thrusters. For this work, the primary focus is Virginia Tech's Ribcraft USV and NPS's SeaFox USV, which are being used to support the development of perception, mapping, and guidance algorithms for riverine USVs [1]. Because the vehicles must operate fully autonomously in a dynamic, uncertain environment, it is essential that their control systems adequately account for vehicle dynamics in planning and executing motions. To broaden the class of vehicles that are considered, we also include system identification results for a low-cost pontoon-based USV that uses differential electric thrusters for propulsion and steering. This technical report is an extended version of [12].

\section{PAGE 1}




\section{Dynamic Modeling}

Hydrodynamicists characterize surface vessel performance in terms of the Froude number $\mathrm{Fr}=U / \sqrt{L g}$, where $U$ is the operating speed, $L$ is the length of the submerged portion of the hull, and $g$ is the acceleration due to gravity. As Faltinsen [4] explains, the weight of a vessel operating with a Froude number less than about 0.4 to 0.5 is almost completely supported by the hydrostatic force of buoyancy that is proportional to the volume of fluid displaced by the vessel. In such a condition, a vessel is said to operate in displacement mode. At the other extreme, the weight of a vessel operating with a Froude number greater than about 1.0 to 1.2 is almost completely supported by a hydrodynamic force that is roughly proportional to the square of the speed. In this condition, a vessel is said to operate in planing mode. For intermediate values of the Froude number, the vessel's weight is supported by both the hydrostatic and hydrodynamic force, resulting in a semi-displacement vessel. Two of the three USVs considered in this paper are capable of operating in planing mode and, indeed, are designed specifically to do so. In practice, though, we expect these vessels may routinely operate in any of the three modes. A successful modeling effort will adequately capture the variations in vehicle performance over the full range of achievable Froude numbers.

With fully instrumented vehicles available for testing, we approach model identification as an experimental problem in which input and state histories are used to determine model parameters. We assume that the USV will operate in a benign environment while model identification data are being collected. For example, we assume that there are no disturbances, such as currents or wind. We also assume that the vehicle moves only in three degrees of freedom: surge, sway, and heave. So, for example, there are no wave disturbances to alter the vehicle's pitch and roll attitude or to excite heave motion. In addition, thrust line effects are ignored; we assume that the force of propulsion induces no pitch or roll moment. (In reality, the propulsor on a turning high-speed vessel will typically induce a roll moment that "banks" the vehicle into the turn. Although we ignore this rolling motion, we do seek a planar motion model that realistically captures turning performance.) Though we anticipate scenarios in which a USV must move in reverse, we consider only forward motion in this technical report.

Beginning from a nonlinear three degree of freedom planar motion model, with force and moment inputs, we make several simplifying assumptions to obtain low order models suitable for identification from experimental data. Important actuator nonlinearities, such as amplitude and rate saturation, are incorporated when identifying parameter values for these simple models.

\subsection{Notation and Reference Frames}

Consider an unmanned surface vehicle (USV) modeled as a rigid hull with an articulated outboard motor. Define a body-fixed reference frame whose $x$-axis (denoted by the unit vector $\boldsymbol{b}_{x}$ ) points forward along the longitudinal axis of the boat, whose $y$-axis (denoted by the unit vector $\boldsymbol{b}_{y}$ ) points to starboard, and whose $z$-axis (denoted $\boldsymbol{b}_{z}=\boldsymbol{b}_{x} \times \boldsymbol{b}_{y}$ ) points

PAGE 2

Virginia Center for Autonomous Systems Report No. 2010-01 
downward, completing the orthonormal triad. We assume that the USV is symmetric about its longitudinal plane. Under this assumption, if the body frame were fixed at the center of gravity, one would find the inertia tensor takes the form

$$
\boldsymbol{I}_{\mathrm{cg}}=\left(\begin{array}{ccc}
I_{\operatorname{cg}_{x x}} & 0 & -I_{\mathrm{cg}_{x z}} \\
0 & I_{\operatorname{cg}_{y y}} & 0 \\
-I_{\mathrm{cg}_{x z}} & 0 & I_{\mathrm{cg}_{z z}}
\end{array}\right)
$$

Following [5], we instead translate the origin of the body reference frame to the point in the $x-z$ plane for which the cross-product of inertia $I_{x z}$ is identically zero. In this body-fixed reference frame, the center of gravity will be located at some point

$$
\boldsymbol{r}_{\mathrm{cg}}=\left(\begin{array}{c}
x_{\mathrm{cg}} \\
0 \\
z_{\mathrm{cg}}
\end{array}\right)
$$

such that, according to the parallel axis theorem,

$$
I_{x z}=0=I_{\mathrm{cg}_{x z}}-m r_{c g_{x}} r_{c g_{z}} .
$$

In this reference frame, the inertia tensor is diagonal:

$$
\boldsymbol{I}=\operatorname{diag}\left(I_{x x}, I_{y y}, I_{z z}\right)
$$

Suppose that the vector $\boldsymbol{x}$ denotes the position of the body coordinate origin with respect to some inertially fixed reference frame, denoted by the unit vectors $\boldsymbol{i}_{x}, \boldsymbol{i}_{y}$, and $\boldsymbol{i}_{z}$. Let $\boldsymbol{R}$ denote the proper rotation matrix which aligns the body coordinate axes with those of the inertial frame. Let the body vector $\boldsymbol{v}=[u, v, w]^{T}$ represent the translational velocity of the body reference frame with respect to the inertial reference frame. Similarly, let $\boldsymbol{\omega}=[p, q, r]^{T}$ represent the body angular rate relative to the inertial reference frame. The rigid body kinematic equations are

$$
\begin{aligned}
\dot{\boldsymbol{x}} & =\boldsymbol{R} \boldsymbol{v} \\
\dot{\boldsymbol{R}} & =\boldsymbol{R} \hat{\boldsymbol{\omega}}
\end{aligned}
$$

where the notation $\hat{\imath}$ denotes the $3 \times 3$ skew-symmetric matrix satisfying $\hat{\boldsymbol{a}} \boldsymbol{b}=\boldsymbol{a} \times \boldsymbol{b}$ for vectors $\boldsymbol{a}$ and $\boldsymbol{b}$. These equations relate the body's translational and rotational velocity to the rate of change of position and attitude. Equation (2) is more typically expressed in coordinates, as three ODE's for the three Euler angles $\phi, \theta$, and $\psi$.

Using equations (1) and (2) the 6 degree of freedom kinematics in the absence of currents become

$$
\left(\begin{array}{c}
\dot{x} \\
\dot{y} \\
\dot{z} \\
\dot{\phi} \\
\dot{\theta} \\
\dot{\psi}
\end{array}\right)=\left(\begin{array}{cccccc}
\mathrm{c} \psi \mathrm{c} \theta & -\mathrm{s} \psi \mathrm{c} \phi+\mathrm{c} \psi \mathrm{s} \theta \mathrm{s} \phi & \mathrm{s} \phi \mathrm{s} \psi+\mathrm{c} \psi \mathrm{c} \phi \mathrm{s} \theta & 0 & 0 & 0 \\
\mathrm{~s} \psi \mathrm{c} \theta & \mathrm{c} \psi \mathrm{c} \phi+\mathrm{s} \phi \mathrm{s} \theta \mathrm{s} \psi & -\mathrm{c} \psi \mathrm{s} \phi+\mathrm{s} \theta \mathrm{s} \psi \mathrm{c} \phi & 0 & 0 & 0 \\
-\mathrm{s} \theta & \mathrm{c} \theta \mathrm{s} \phi & \mathrm{c} \theta \mathrm{c} \phi & 0 & 0 & 0 \\
0 & 0 & 0 & 1 & \mathrm{~s} \phi \mathrm{t} \theta & \mathrm{c} \phi \mathrm{t} \theta \\
0 & 0 & 0 & 0 & \mathrm{c} \phi & -\mathrm{s} \phi \\
0 & 0 & 0 & 0 & \mathrm{~s} \phi / \mathrm{c} \theta & \mathrm{c} \phi / \mathrm{c} \theta
\end{array}\right)\left(\begin{array}{c}
u \\
v \\
w \\
p \\
q \\
r
\end{array}\right)
$$

\section{PAGE 3}



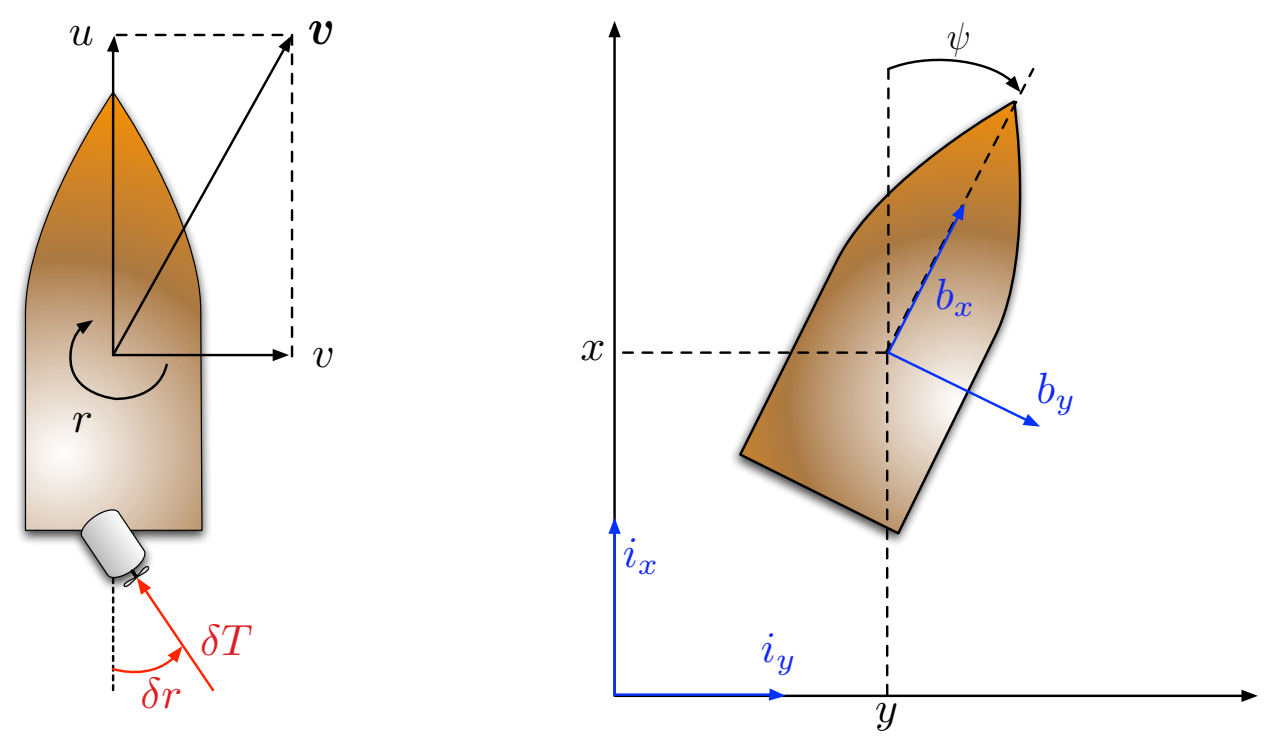

Figure 1: Schematic depiction of a USV.

with $\mathrm{c}=\cos , \mathrm{s}=\sin$, and $\mathrm{t}=\tan$.

To simplify matters, we will ignore the roll, pitch, and heave dynamics and consider USV motion in the horizontal plane. This reformulation leads to ignoring planing dynamics, implying that the boat's dynamics cannot be modeled with constant parameters through the entire performance envelope. Different planing situations can be recovered by having different added mass values for each planing mode. Figure 1 illustrates the state and input variables for a planar USV model with a gimbaled thruster (e.g., an outboard engine).

The kinematic model for a USV in planar motion in the absence of currents is

$$
\left(\begin{array}{l}
\dot{x} \\
\dot{y} \\
\dot{\psi}
\end{array}\right)=\left(\begin{array}{ccc}
\cos \psi & -\sin \psi & 0 \\
\sin \psi & \cos \psi & 0 \\
0 & 0 & 1
\end{array}\right)\left(\begin{array}{l}
u \\
v \\
r
\end{array}\right)
$$

We define the sideslip angle $\beta$, the course angle $\chi$, and the total velocity $V$ as follows:

$$
\beta=\arctan \left(\frac{v}{u}\right), \quad \chi=\psi+\beta, \quad \text { and } \quad V=\sqrt{u^{2}+v^{2}}
$$

Note that the course angle and the heading angle are equal unless the vehicle is slipping. These definitions are illustrated in Figure 2. Recognizing that

$$
\left(\begin{array}{l}
u \\
v
\end{array}\right)=\left(\begin{array}{cc}
\cos \beta & -\sin \beta \\
\sin \beta & \cos \beta
\end{array}\right)\left(\begin{array}{l}
V \\
0
\end{array}\right),
$$

we may write

$$
\left(\begin{array}{l}
\dot{x} \\
\dot{y} \\
\dot{\psi}
\end{array}\right)=\left(\begin{array}{ccc}
\cos \psi & -\sin \psi & 0 \\
\sin \psi & \cos \psi & 0 \\
0 & 0 & 1
\end{array}\right)\left(\begin{array}{ccc}
\cos \beta & -\sin \beta & 0 \\
\sin \beta & \cos \beta & 0 \\
0 & 0 & 1
\end{array}\right)\left(\begin{array}{l}
V \\
0 \\
r
\end{array}\right)
$$

PAGe 4 
or

$$
\left(\begin{array}{c}
\dot{x} \\
\dot{y} \\
\dot{\chi}
\end{array}\right)=\left(\begin{array}{ccc}
\cos \chi & -\sin \chi & 0 \\
\sin \chi & \cos \chi & 0 \\
0 & 0 & 1
\end{array}\right)\left(\begin{array}{c}
V \\
0 \\
r+\dot{\beta}
\end{array}\right) \cdot
$$

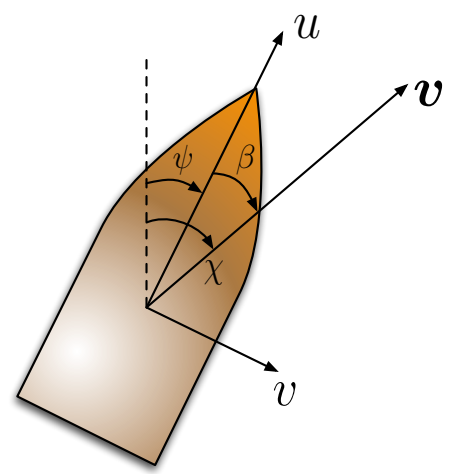

Figure 2: Definitions of sideslip and course angles.

\subsection{Nonlinear Planar Model}

The dynamics of a boat can be described, using the notation

$$
\begin{aligned}
& \boldsymbol{\nu}=\left[\begin{array}{lll}
u & v & r
\end{array}\right]^{T} \\
& \boldsymbol{\eta}=\left[\begin{array}{lll}
x & y & \psi
\end{array}\right]^{T}
\end{aligned}
$$

as,

$$
M \dot{\nu}+C(\nu) \nu+D(\nu) \nu=f
$$

where $\boldsymbol{M}$ is the mass matrix, $\boldsymbol{C}$ is the Coriolis and centripetal matrix, $\boldsymbol{D}$ is the damping matrix, and $\boldsymbol{f}$ are the control forces and moments. The mass matrix $\boldsymbol{M}$ and Coriolis and centripetal matrix $\boldsymbol{C}$ can be further decomposed into

$$
\boldsymbol{M}=\boldsymbol{M}_{\mathrm{RB}}+\boldsymbol{M}_{\mathrm{A}} \quad \text { and } \quad \boldsymbol{C}=\boldsymbol{C}_{\mathrm{RB}}+\boldsymbol{C}_{\mathrm{A}}
$$

which are composed of rigid body contributions (e.g., the mass and moments of inertia) and contributions due to the fluid (e.g., the "added mass"). In general, for a surface vehicle, added mass effects will depend on the wave motion and other disturbances. We neglect such variations in added mass and inertia and assume that the mass matrix takes the following form

$$
\boldsymbol{M}=\left(\begin{array}{ccc}
m-X_{\dot{u}} & 0 & 0 \\
0 & m-Y_{\dot{v}} & m x_{G}-N_{\dot{v}} \\
0 & m x_{G}-N_{\dot{v}} & I_{z}-N_{\dot{r}}
\end{array}\right)
$$

where the added mass and inertia parameters are constant, at least when the vehicle is operating near a given nominal motion. 
The Coriolis and centripetal matrix is

$$
\boldsymbol{C}(\boldsymbol{\nu})=\left(\begin{array}{ccc}
0 & 0 & -m\left(x_{G} r+v\right)+Y_{\dot{v}} v+N_{\dot{v}} r \\
0 & 0 & \left(m-X_{\dot{u}}\right) u \\
m\left(x_{G} r+v\right)-Y_{\dot{v}} v-N_{\dot{v}} r & \left(X_{\dot{u}}-m\right) u & 0
\end{array}\right)
$$

The damping matrix is composed of a linear damping matrix and quadratic damping matrix such that

$$
\boldsymbol{D}(\boldsymbol{\nu})=\boldsymbol{D}_{l}+\boldsymbol{D}_{q}(\boldsymbol{\nu})
$$

The linear damping matrix arises due to laminar friction on the body of the boat. Forward velocity is assumed to decouple from turn rate and side slip velocity due to port to starboard symmetry. Also assuming planar motion, the linear damping matrix takes the form:

$$
\boldsymbol{D}_{l}=-\left(\begin{array}{ccc}
X_{u} & 0 & 0 \\
0 & Y_{v} & Y_{r} \\
0 & N_{v} & N_{r}
\end{array}\right)
$$

The quadratic damping matrix comes about due to vortex shedding off of the hull of the boat. This effect is similar to the drag due to lift for aircraft. Assuming planar motion and port to starboard symmetry, which decouples forward velocity with side slip velocity and turn rate, the quadratic damping matrix takes the form:

$$
\boldsymbol{D}_{q}(\boldsymbol{\nu})=\left(\begin{array}{ccc}
X_{u|u|}|u| & 0 & 0 \\
0 & Y_{v|v|}|v| & Y_{r|r|}|r| \\
0 & N_{v|v|}|v| & N_{r|r|}|r|
\end{array}\right)
$$

Combining these two damping matrices yields

$$
\boldsymbol{D}(\boldsymbol{\nu})=\left(\begin{array}{ccc}
X_{u}+X_{u|u|}|u| & 0 & 0 \\
0 & Y_{v}+Y_{v|v|}|v| & Y_{r}+Y_{r|r|}|r| \\
0 & N_{v}+N_{v|v|}|v| & N_{r}+N_{r|r|}|r|
\end{array}\right)
$$

For a USV with inputs taking the form of generalized forces and moments based upon throttle and steering angle, the equations of motion are:

$$
\begin{aligned}
m\left(\dot{u}-v r-x_{\mathrm{cg}} r^{2}\right) & =X_{\dot{u}} \dot{u}+X_{u} u+X_{|u| u}|u| u+X_{r r} r^{2}+X_{v r} v r+X_{\mathrm{ctrl}}(\delta r, \delta T) \\
m\left(\dot{v}-u r-x_{\mathrm{cg}} \dot{r}\right) & =Y_{\dot{v}} \dot{v}+Y_{\dot{r}} \dot{r}+Y_{v} v+Y_{r} r+Y_{|v| v \mid}\left|v+Y_{|r| r}\right| r \mid r+Y_{\mathrm{ctrl}}(\delta r, \delta T) \\
I_{z z} \dot{r}+m x_{\mathrm{cg}}(\dot{v}+u r) & =N_{\dot{v}} \dot{v}+N_{\dot{r}} \dot{r}+N_{v} v+N_{r} r+N_{|v| v}|v| v+N_{|r| r}|r| r+N_{\mathrm{ctrl}}(\delta r, \delta T)
\end{aligned}
$$

In these equations, the propulsor generates a surge force $X_{\text {ctrl }}$ which may, in general, be a complicated function of the vehicle state as well as throttle setting $\delta T$ (measured in percent) and the steering angle $\delta r$. Likewise, the steering moment $N_{\text {ctrl }}(\delta r, \delta T)$ and the side force $Y_{\text {ctrl }}(\delta r, \delta T)$ resulting from control deflections may also depend on the full state. Anticipating the use of simplified models that are identifiable from limited data sets, we do not explicitly incorporate this state-dependence into the force and moment model.

In the following sections, we present several simplified variants of these dynamic equations. Again, our objective is to obtain a model that is rich enough to enable effective motion planning and control, simple enough to allow quick identification, and general enough to describe a variety of vehicles operating over a large range of speeds.

\section{PAGe 6}

Virginia Center for Autonomous Systems Report No. 2010-01 


\subsection{Linear Models}

\subsubsection{Speed Dynamics}

Under the stated assumptions, equation (14) gives the following nonlinear speed dynamics

$$
m\left(\dot{u}-v r-x_{\mathrm{cg}} r^{2}\right)=X_{\dot{u}} \dot{u}+X_{u} u+X_{|u| u}|u| u+X_{r r} r^{2}+X_{v r} v r+X_{\mathrm{ctrl}}(\delta r, \delta T)
$$

Corresponding to a given nominal thrust value $X_{\operatorname{ctrl}_{0}}$, is a steady speed $u_{0}$. Linearizing about the steady motion corresponding to the state variable values

$$
u=u_{0}, \quad v=0, \quad \text { and } \quad r=0,
$$

one obtains the perturbation dynamics

$$
\left(m-X_{\dot{u}}\right) \Delta \dot{u}=X_{u} \Delta u+\Delta X_{\text {ctrl }}
$$

As already mentioned, the thrust $X_{\text {ctrl }}$ may, in general, be a complicated function of the vehicle state as well as the throttle setting and steering angle. While dynamic propulsion models do exist and could, in principle, be identified through careful experimentation, we do not explicitly account for thruster dynamics. Rather, we identify the following first order model relating throttle setting to vehicle speed:

$$
\tau_{u} \Delta \dot{u}+\Delta u=K_{u} \delta T, \quad u=u_{0}+\Delta u
$$

While this linear first order model represents a standard approach, alternate models have been proposed. Breivik and coauthors, for example, proposed a nonlinear variation of this model in [3]. The model can be put into state-space form

$$
\Delta \dot{u}=a \Delta u+b \Delta \delta T
$$

with parameters $a$ and $b$.

\subsubsection{Steering Dynamics}

Linearized Steering Dynamics. Linearizing (15) and (16) about the steady motion corresponding to the state variable values

$$
u=u_{0}, \quad v=0, \quad \text { and } \quad r=0,
$$

and the nominal thrust value $T_{0}$, and assuming that $\sin \delta \approx \delta$, one obtains the following linear system

$$
\left(\begin{array}{cc}
m-Y_{\dot{v}} & m x_{\mathrm{cg}}-Y_{\dot{r}} \\
m x_{\mathrm{cg}}-N_{\dot{v}} & I_{z z}-N_{\dot{r}}
\end{array}\right)\left(\begin{array}{c}
\dot{v} \\
\dot{r}
\end{array}\right)+\left(\begin{array}{cc}
-Y_{v} & m u_{0}-Y_{r} \\
-N_{v} & m x_{\mathrm{cg}} u_{0}-N_{r}
\end{array}\right)\left(\begin{array}{c}
v \\
r
\end{array}\right)=\left(\begin{array}{c}
Y_{\delta r} \\
N_{\delta r}
\end{array}\right) \delta r
$$

Recall that the stability derivatives $Y_{v}, Y_{r}, Y_{\delta}, N_{v}, N_{r}$, and $N_{\delta}$ are parameterized by the nominal surge velocity $u_{0}$. Because the sway and yaw dynamics decouple from the speed 
dynamics in the linearization, the linearized model suggests that there is no first order effect of small changes in thrust on the sway and yaw dynamics. (Of course, this is a direct consequence of the assumed state-dependencies of $X, Y$, and $N$.) The model can be put into state-space form

$$
\left(\begin{array}{c}
\dot{v} \\
\dot{r}
\end{array}\right)=\boldsymbol{A}\left(\begin{array}{c}
v \\
r
\end{array}\right)+\boldsymbol{B} \delta r
$$

with parameters $\boldsymbol{A}$ and $\boldsymbol{B}$.

Nomoto's Steering Models. Considering large displacement vessels, Nomoto and colleagues [10] suggested that turn rate can be described as a simple first order equation relating rudder angle, $\delta$, and turn rate, $r$, at a given forward speed $u_{0}$.

$$
\tau \dot{r}+r=K_{r} \delta
$$

Adding an integrator cascade to incorporate heading information,

$$
\left[\begin{array}{c}
\dot{\psi} \\
\dot{r}
\end{array}\right]=\left(\begin{array}{cc}
0 & 1 \\
0 & -\frac{1}{\tau_{r}}
\end{array}\right)\left[\begin{array}{l}
\psi \\
r
\end{array}\right]+\left[\begin{array}{c}
0 \\
\frac{K_{r}}{\tau_{r}}
\end{array}\right] \delta r
$$

Nomoto's model assumes that the boat is constrained to move wherever it is pointed; it cannot skid while turning. Boats that steer with vectored thrust and a rudder must create a sideslip angle, $\beta$, between the nose of the boat and the velocity vector relative to the water in order to provide a force pushing the boat to the inside of the turn. The linear steering model intrinsically allows for sideslip. To address this deficiency in the Nomoto model, without resorting to the full linearized steering model (20), it is natural to modify Nomoto's model to accommodate a first order lag for sideslip angle, $\beta$ :

$$
\tau_{\beta} \dot{\beta}+\beta=-K_{\beta} r
$$

called Nomoto's model with sideslip [14]. Incorporating this equation in (23) gives

$$
\left[\begin{array}{c}
\dot{\beta} \\
\dot{\psi} \\
\dot{r}
\end{array}\right]=\left(\begin{array}{ccc}
-\frac{1}{\tau_{\beta}} & 0 & -\frac{K_{\beta}}{\tau_{\beta}} \\
0 & 0 & 1 \\
0 & 0 & -\frac{1}{\tau_{r}}
\end{array}\right)\left[\begin{array}{l}
\beta \\
\psi \\
r
\end{array}\right]+\left[\begin{array}{c}
0 \\
0 \\
\frac{K_{r}}{\tau_{r}}
\end{array}\right] \delta r
$$

\subsection{Low-Speed Nonlinear Model}

At very low speeds, potential flow theory provides a good description of the dominant hydrodynamic effects. The following nonlinear model describes the planar motion of a rigid spheroid immersed in a perfect fluid [7]:

$$
\begin{aligned}
m(\dot{u}-v r) & =X_{\dot{u}} \dot{u}-Y_{\dot{v}} v r \\
m(\dot{v}-u r) & =Y_{\dot{v}} \dot{v}+X_{\dot{u}} u r \\
I_{z z} \dot{r} & =N_{\dot{r}} \dot{r}+\left(X_{\dot{u}}-Y_{\dot{v}}\right) u v
\end{aligned}
$$

PAGE 8

Virginia Center for Autonomous Systems Report No. 2010-01 
This model is a well-used example of an underactuated mechanical system, appearing in a variety of papers on motion planning and control for such systems. Incorporating inputs and decoupled linear damping terms gives

$$
\begin{aligned}
m(\dot{u}-v r) & =X_{\dot{u}} \dot{u}-Y_{\dot{v}} v r+X_{u|u|} u|u|+X_{\mathrm{ctrl}}(\delta r, \delta T) \\
m(\dot{v}-u r) & =Y_{\dot{v}} \dot{v}+X_{\dot{u}} u r+Y_{v|v|} v|v|+Y_{\mathrm{ctrl}}(\delta r, \delta T) \\
I_{z z} \dot{r} & =N_{\dot{r}} \dot{r}+\left(X_{\dot{u}}-Y_{\dot{v}}\right) u v+N_{r|r|} r|r|+N_{\mathrm{ctrl}}(\delta r, \delta T)
\end{aligned}
$$

The nonlinear model (26) is a crude approximation of the dynamics derived in Section 2.2, shown in equations (14) through (16), but the relatively few model parameters may be identified from simple field tests. Notice that the inputs are included in a general way to allow for use with multiple actuator configurations. In addition to studying the speedparameterized linear models described in Section 2.3, we investigate whether (26) can better model a USV's behavior at low speeds. The model can be put in the state-space form

$$
\left(\begin{array}{c}
\dot{u} \\
\dot{v} \\
\dot{r}
\end{array}\right)=\boldsymbol{A}\left(\begin{array}{c}
v r \\
u r \\
u v \\
u|u| \\
v|v| \\
r|r|
\end{array}\right)+\boldsymbol{B} \boldsymbol{\phi}_{B}(\delta r, \delta T)
$$

\section{Model Identification}

Model identification proceeds in two steps. The first step is to determine the steady-state relationship between inputs and outputs from open-loop maneuvers. With the "DC gains" determined, the second step is to identify the parameters that govern transient behavior. Inspired by previous system identification efforts for autonomous underwater vehicles [11], we implement simple controllers for motion variables of interest (e.g., speed and heading) and obtain closed-loop state histories corresponding to specified, desired output histories (e.g., a square wave in heading angle). Using closed-loop system responses instead of open-loop responses allows more control over the vehicle's motion in the confined operating environments where field tests are performed. Including control dynamics (such as a PID controller) potentially reveals previously hidden vehicle behaviors.

Our approach to model identification involves adapting model parameter values such that a model-based simulation best matches the experimental data obtained for the feedback controlled USV. To initialize this iterative identification procedure, we obtain approximate values for the dynamic parameters using a least squares fit to the experimental data. Once an initial estimate for the parameter values has been obtained, the identification method seeks to minimize a measure of the error between the true and simulated state and control histories. Let $\nu_{i}(t)$ represent a particular state or input for the experimental system, , nondimensionalized using some characteristic values of mass, distance, and time. Let $\hat{\nu}_{i}$ represent the corresponding nondimensional state or input for the simulated mathematical model. For

\section{PAGE 9}

Virginia Center for Autonomous Systems Report No. 2010-01 
given experimental and simulated state and input histories, one might seek model parameter values which minimize a cost function

$$
J=\left[\frac{1}{T} \int_{0}^{T} \sum_{i}\left\|\nu_{i}(t)-\hat{\nu}_{i}(t)\right\|^{2} d t\right]^{\frac{1}{2}}
$$

The question of identifiability naturally arises. For linear dynamic models, as discussed in [11], it is sufficient to verify that the system's Markov parameters depend appropriately on the parameters to be identified [6]. For the nonlinear dynamic model, one may consider local identifiability by linearizing around a nominal state.

\subsection{Identifying Steady-State Input/Output Relationships}

To determine the steady-state gains for the linear models described in Section 2.3, one simply applies a constant input and measures the steady-state output. For the linear speed model, for example, one applies a step input in throttle and measures the resulting steadystate deviation from the initial, nominal speed. For the linear steering model, at some given, constant operating speed, one applies a step input in steering angle and measures the resulting steady-state turn rate and side velocity. This is repeated for a range of step inputs in steering angle. The process for constructing steady-state mappings from input to output for the nonlinear slow speed model shown in Section 2.4 is similar.

For throttle settings above idle (above about 30\% for the Ribcraft USV), steady-state speed increases with throttle setting and decreases with steering angle. The steady-state turn rate and side velocity also increase with throttle setting for nonzero steering angles. We assume the following mappings:

$$
\underbrace{\left(\begin{array}{c}
u_{\mathrm{ss}} \\
v_{\mathrm{ss}} \\
r_{\mathrm{ss}}
\end{array}\right)}_{\boldsymbol{x}_{\mathrm{ss}}}=\underbrace{\left(\begin{array}{ccc}
u_{\mathrm{bias}} & u_{\delta T} & u_{\delta \mathrm{T}, \delta \mathrm{r}} \\
0 & 0 & v_{\delta \mathrm{T}, \delta \mathrm{r}} \\
0 & 0 & r_{\delta \mathrm{T}, \delta \mathrm{r}}
\end{array}\right)}_{\boldsymbol{K}} \underbrace{\left(\begin{array}{c}
1 \\
\delta T \\
\delta T \delta r
\end{array}\right)}_{\boldsymbol{\phi}_{u}\left(\boldsymbol{u}_{\mathrm{ss}}\right)}
$$

which is in the form

$$
\boldsymbol{x}_{\mathrm{ss}}=\boldsymbol{K} \phi_{u}\left(\boldsymbol{u}_{\mathrm{ss}}\right)
$$

Above 30\% throttle for the Ribcraft USV, steady-state forward velocity begins to vary linearly with throttle over a range of constant steering angles. Define "forward velocity reduction" as the decrease in forward velocity when turning. Experimental results suggest that, for a constant throttle setting, the forward velocity reduction is linear in the steering angle. Also, for increasing values of the constant throttle setting, the forward velocity reduction increases. Note that there are nonlinear actuator phenomena at throttle between $0 \%$ and $30 \%$. For the Ribcraft USV, for example, forward motion resulting from throttle settings

less than $30 \%$ is the same as forward motion resulting from a $30 \%$ throttle setting. Zero throttle results in the boat sitting at rest. Thus, polynomial fits of experimental data to equations (28) are only valid above the $30 \%$ throttle setting. 


\subsection{Enforcing Steady-State Input/Output Relationships When Iden- tifying Dynamic Parameters}

Each of the USV dynamic models presented in Sections 2.3 and 2.4 can be represented in the form

$$
\dot{\boldsymbol{x}}=\boldsymbol{A} \boldsymbol{\phi}_{A}(\boldsymbol{x})+\boldsymbol{B} \boldsymbol{\phi}_{B}(\boldsymbol{u})
$$

where $\boldsymbol{x}$ represents the state vector and $\boldsymbol{u}$ represents the input vector. The components of the vector $\boldsymbol{B} \phi_{B}(\boldsymbol{u})$ model the force and moment generated by a given throttle and steering command. (Recall that we have assumed the control force and moment are static functions of the control parameters $\delta r$ and $\delta T$.) The vector $\boldsymbol{\phi}(\boldsymbol{x})_{A}$ represents the dependency of $\dot{\boldsymbol{x}}$ on the state. The vector $\boldsymbol{\phi}(\boldsymbol{x})_{B}$ represents the dependency of $\dot{\boldsymbol{x}}$ on the inputs. While this dependency may be nonlinear for $\boldsymbol{\phi}(\boldsymbol{x})_{A}$ and $\boldsymbol{\phi}(\boldsymbol{u})_{B}$, they are linearly parameterized by the matrices $\boldsymbol{A}$ and $\boldsymbol{B}$ respectively. Also, assume that the nonlinearities arising in $\boldsymbol{\phi}(\boldsymbol{x})_{A}$ and $\phi(\boldsymbol{u})_{B}$ are due to multiplications of the states and inputs respectively. The parameters contained in $\boldsymbol{A}$ are dynamic parameters that remain to be determined and $\boldsymbol{B}(\boldsymbol{A})$. Because the vessel dynamics are stable, a constant input $\boldsymbol{u}_{\mathrm{ss}}$ ultimately yields the steady-state relationship

$$
\mathbf{0}=\boldsymbol{A} \phi_{A}\left(\boldsymbol{x}_{\mathrm{ss}}\right)+\boldsymbol{B} \phi_{B}\left(\boldsymbol{u}_{\mathrm{ss}}\right) .
$$

with $\boldsymbol{x}_{\mathrm{ss}}$ and $\boldsymbol{u}_{\mathrm{ss}}$ being steady-state constants. Using equation (29) we have

$$
\boldsymbol{B} \phi_{B}\left(\boldsymbol{u}_{\mathrm{ss}}\right)=-\boldsymbol{A} \phi_{A}\left(\boldsymbol{K} \boldsymbol{\phi}_{\boldsymbol{u}}\left(\boldsymbol{u}_{\mathrm{ss}}\right)\right)
$$

The dynamic parameters, defining the matrix $\boldsymbol{A}$, are the variables being adjusted in the identification process. The matrix $\boldsymbol{B}$ is defined by $\boldsymbol{A}, \boldsymbol{K}$, and $\boldsymbol{u}_{\mathrm{ss}}$.

To understand how the steady-state mappings from inputs to outputs, described in Section 3.1, constrain the model identification process, consider the following 2-input/2-output system as an example.

$$
\underbrace{\left(\begin{array}{c}
\dot{x}_{1} \\
\dot{x}_{2}
\end{array}\right)}_{\boldsymbol{x}}=\underbrace{\left(\begin{array}{lll}
a_{11} & a_{12} & a_{13} \\
a_{21} & a_{22} & a_{23}
\end{array}\right)}_{\boldsymbol{A}} \underbrace{\left(\begin{array}{c}
x_{1} \\
x_{2} \\
x_{1} x_{2}
\end{array}\right)}_{\boldsymbol{\phi}_{A}}+\boldsymbol{B} \boldsymbol{\phi}_{B}(\boldsymbol{u})
$$

where the undetermined terms defining $\boldsymbol{B} \boldsymbol{\phi}_{B}(\boldsymbol{u})$ must satisfy some required steady-state behavior

$$
\begin{aligned}
& x_{1_{\mathrm{ss}}}=\alpha_{1} u_{1_{\mathrm{ss}}}+\alpha_{2} u_{2_{\mathrm{ss}}}^{2} \\
& x_{2_{\mathrm{ss}}}=\alpha_{3} u_{2_{\mathrm{ss}}}
\end{aligned}
$$

where the constant coefficients $\alpha_{i}$ that correspond to (29) have been determined, for example, from analysis of experimental data. 
Using (31) and the steady-state mappings in (34) and (35), we find that

$$
\begin{aligned}
\boldsymbol{B} \phi_{B}(\boldsymbol{u}) & =\left(\begin{array}{l}
-a_{11} x_{1_{\mathrm{ss}}}-a_{12} x_{2_{\mathrm{ss}}}-a_{13} x_{1_{\mathrm{ss}}} x_{2_{\mathrm{ss}}} \\
-a_{21} x_{1_{\mathrm{ss}}}-a_{22} x_{2_{\mathrm{ss}}}-a_{23} x_{1_{\mathrm{ss}}} x_{2_{\mathrm{ss}}}
\end{array}\right) \\
& =\left(\begin{array}{l}
-a_{11}\left(\alpha_{1} u_{1_{\mathrm{ss}}}+\alpha_{2} u_{2_{\mathrm{ss}}}^{2}\right)-a_{12} \alpha_{3} u_{2_{\mathrm{ss}}}-a_{13}\left(\alpha_{1} \alpha_{3} u_{1_{\mathrm{ss}}} u_{2_{\mathrm{ss}}}+\alpha_{3} \alpha_{2} u_{2_{\mathrm{ss}}}^{3}\right) \\
-a_{21}\left(\alpha_{1} u_{1_{\mathrm{ss}}}+\alpha_{2} u_{2_{\mathrm{ss}}}^{2}\right)-a_{22} \alpha_{3} u_{2_{\mathrm{ss}}}-a_{23}\left(\alpha_{1} \alpha_{3} u_{1_{\mathrm{ss}}} u_{2_{\mathrm{ss}}}+\alpha_{3} \alpha_{2} u_{2_{\mathrm{ss}}}^{3}\right)
\end{array}\right)
\end{aligned}
$$

Dropping the subscript "ss" and re-arranging gives

$$
\boldsymbol{B} \boldsymbol{\phi}_{B}(\boldsymbol{u})=\underbrace{\left(\begin{array}{ccccc}
-a_{11} \alpha_{1} & -a_{12} \alpha_{3} & -a_{11} \alpha_{2} & -a_{13} \alpha_{2} \alpha_{3} & -a_{13} \alpha_{1} \alpha_{3} \\
-a_{21} \alpha_{1} & -a_{22} \alpha_{3} & -a_{21} \alpha_{2} & -a_{23} \alpha_{2} \alpha_{3} & -a_{23} \alpha_{1} \alpha_{3}
\end{array}\right)}_{\boldsymbol{B}} \underbrace{\left(\begin{array}{c}
u_{1} \\
u_{2} \\
u_{2}^{2} \\
u_{2}^{3} \\
u_{1} u_{2}
\end{array}\right)}_{\boldsymbol{\phi}(\boldsymbol{u})_{B}}
$$

Thus, given values of $\alpha_{1}, \alpha_{2}$, and $\alpha_{3}$ obtained from steady-state input/output data, one may impose the conditions (37) in the dynamics (33) when identifying the parameters $a_{i j}$. The identification process is thus constrained to yield parameter values that give proper steady-state response.

\subsection{Initializing the Algorithm that Identifies Dynamic Parameters}

Referring to (30), suppose that we have obtained a (dynamic) state history $\boldsymbol{x}(t)$, and the corresponding state rate history $\dot{\boldsymbol{x}}(t)$, in response to some suitably rich input history $\boldsymbol{u}(t)$. Sampling these time histories at specific instants, one obtains a collection of algebraic equations for the unknown matrix $\boldsymbol{A}$ at time instants $t_{i}$, subject to the constraints imposed by equations (31):

$$
\begin{aligned}
& \dot{\boldsymbol{x}}\left(t_{i}\right)=\boldsymbol{A} \boldsymbol{\phi}_{A}\left(\boldsymbol{x}\left(t_{i}\right)\right)+\boldsymbol{B} \phi_{B}\left(\boldsymbol{u}\left(t_{i}\right)\right) \\
& \dot{\boldsymbol{x}}\left(t_{i}\right)=\boldsymbol{A}\left(\phi_{A}\left(\boldsymbol{x}\left(t_{i}\right)\right)-\boldsymbol{\phi}_{A}\left(\boldsymbol{K} \boldsymbol{\phi}_{u}\left(\boldsymbol{u}\left(t_{i}\right)\right)\right)\right.
\end{aligned}
$$

These equations collectively constitute an overdetermined linear system for the unknown parameters in the matrix $\boldsymbol{A}$. The matrix $\hat{\boldsymbol{A}}$ which most closely satisfies the given algebraic equations, in a least-square error sense, can be obtained using the pseudo-inverse [13]. These least squares dynamic parameters are used as a starting point for the optimization algorithm, which defines closed-loop dynamic parameters.

\subsection{Identifying Dynamic Parameters}

Each of the USVs considered here has single-input, single-output PID compensators that adjust rudder and throttle commands to maintain a commanded heading and velocity, respectively. Petrich [11] describes an approach to system identification for closed-loop systems 
with unknown plant parameters in which the identification algorithm attempts to minimize the root-mean-squared (rms) error between true and simulated state histories in response to identical input histories (e.g., square waves of given period and amplitude). This closed loop approach is required to excite potentially unmodeled dynamics. By using the least squares open loop parameters as a seed value in the optimization, these unmodeled dynamics are estimated by a change to the open loop parameters. Each of the proposed models is simulated in Simulink, along with a PID compensator with control gains identical to those used on the actual USV. Input saturation and rate limiting are also incorporated into the Simulink model, to more closely approximate the true vehicle behavior. A Matlab implementation of the the Nelder-Mead simplex method is used to adjust the parameters contained in the matrix $\boldsymbol{A}$ (described in previous sections) with the aim of minimizing a cost function of the form

$$
J=\dot{\chi}_{\mathrm{rms}}+\chi_{\mathrm{rms}}+\delta r_{\mathrm{rms}}+u_{\mathrm{rms}}+\delta T_{\mathrm{rms}}
$$

where

$$
\nu_{\mathrm{rms}}=\sqrt{\frac{1}{T} \int_{0}^{T}\|\nu(t)-\hat{\nu}(t)\|^{2} d t}
$$

for a given variable $\nu \in\{\chi, \dot{\chi}, u, \delta r, \delta T\}$ where $T$ is the duration of the experiment. Each variable is non-dimensionalized with nominal values for velocity, boat length, and throttle setting appropriately. (The cost function might vary with the type of model being considered. For the first order Nomoto model, for example, where speed and steering are decoupled, one would omit $u$ and $\delta T$ from the cost function.)

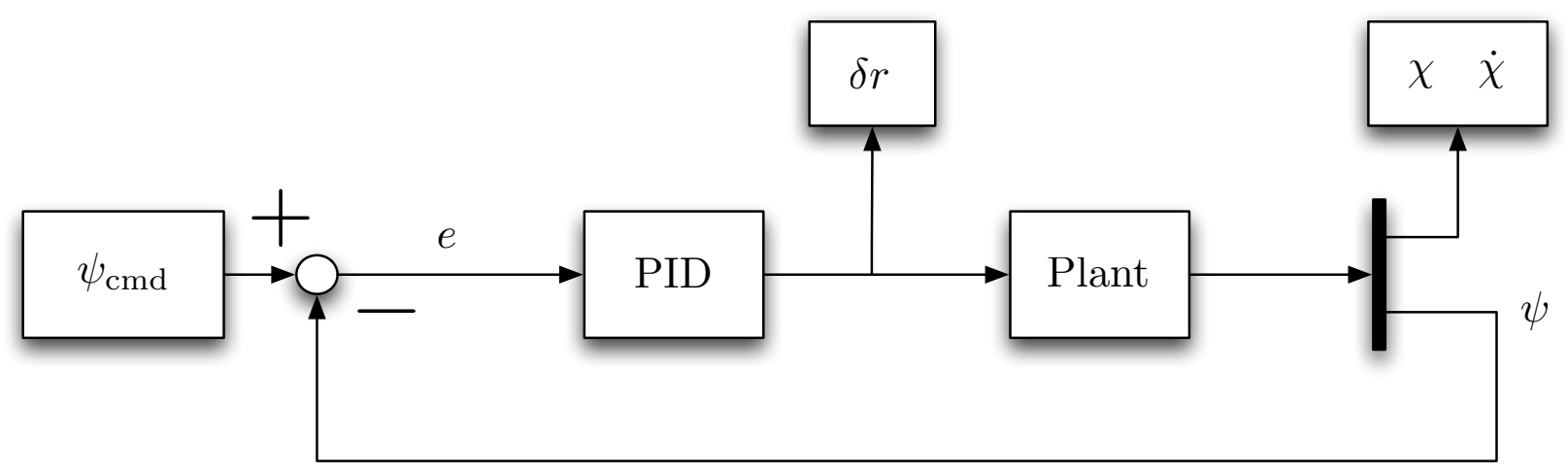

Figure 3: Closed-loop control scheme. Additional signals used in model identification are shown as outputs.

\section{Platforms}

To support the claim that the proposed models are sufficiently general to apply to a variety of hullforms and actuator configurations, we consider three distinct vehicles. The actuator configurations include an outboard motor, a water jet thruster, and dual electric thrusters. Thus, we consider a vectored thruster with rudder (the outboard motor), a vectored thruster 
(the water jet), and differential steering (the dual electric thrusters). Researchers at Virginia Tech have developed and operate two of these USVs (those with an outboard motor and with dual electric thrusters) while researchers at the Naval Postgraduate School operate the water jet propelled USV.

\subsection{Ribcraft USV}

Virginia Tech has modified a Ribcraft 4.8 Professional boat, built in Marblehead, MA, for autonomous operation. The Ribcraft is a 15-foot long, fiberglass-hulled, rigid inflatable boat (RIB) with a 50 horsepower, hydraulically steered Honda outboard motor. The motor can propel the boat to roughly 20 knots.

An Ibeo laser line scanner is the primary visual sensor. The line scanner is mounted to a 2-axis servo-actuated gimbal which is, in turn, mounted to the aft antenna arch. An AHRS mounted on the laser line scanner provides feedback signals for keeping the device level. A DGPS receiver is mounted to the cockpit seat and a vessel AHRS is mounted underneath the seat. The on-board computer processes sensor data and outputs control commands, allowing various levels of control, from simple open-loop steering and throttle commands to closed-loop waypoint following and cross-track control.

The mission scenario which drove the development of this USV is autonomous navigation in a riverine environment with little or no a priori information about the waterway. A high level controller gives cross track control commands via a series of waypoints, but the path can be quickly re-planned when the vehicle encounters unforseen obstructions. The laser line scanner can detect obstacles within a 250 meter radius and within a $150^{\circ}$ field of view. A powerful wireless antenna is mounted to the antenna arch that allows the on-board computer to communicate with a computer on a chase boat. This chase boat computer can send open-loop commands and waypoints. An emergency stop which sets the throttle to idle and stops control of the steering angle is mounted within easy reach of the driver. There is also a wireless emergency stop if the boat is running without occupants. The Ribcraft USV is shown in Figure 4.

\subsection{SeaFox USV}

The NPS Center for Autonomous Vehicle Research (CAVR) operates a SeaFox USV to support research and development of new technologies for increased autonomy in military unmanned vehicles. The NPS SeaFox was designed and manufactured by Northwind Marine (Seattle, WA) as a remote-controlled platform for ISR, anti-terrorism force protection (ATFP), and maritime interdiction operations (MIO) and is shown in Figure 5. SeaFox is a 16-foot long, aluminum-hulled, rigid inflatable boat (RIB) with a 5-foot, 9-inch beam; 10-inch draft; fold-down communications mast; and fully-enclosed electronics and engine compartments. SeaFox's water jet propulsion system is powered by a JP5-fueled, 250-HP V-6 Mercury Racing engine, and can deliver a top speed of 40 knots.

PAGe 14

Virginia Center for Autonomous Systems Report No. 2010-01 


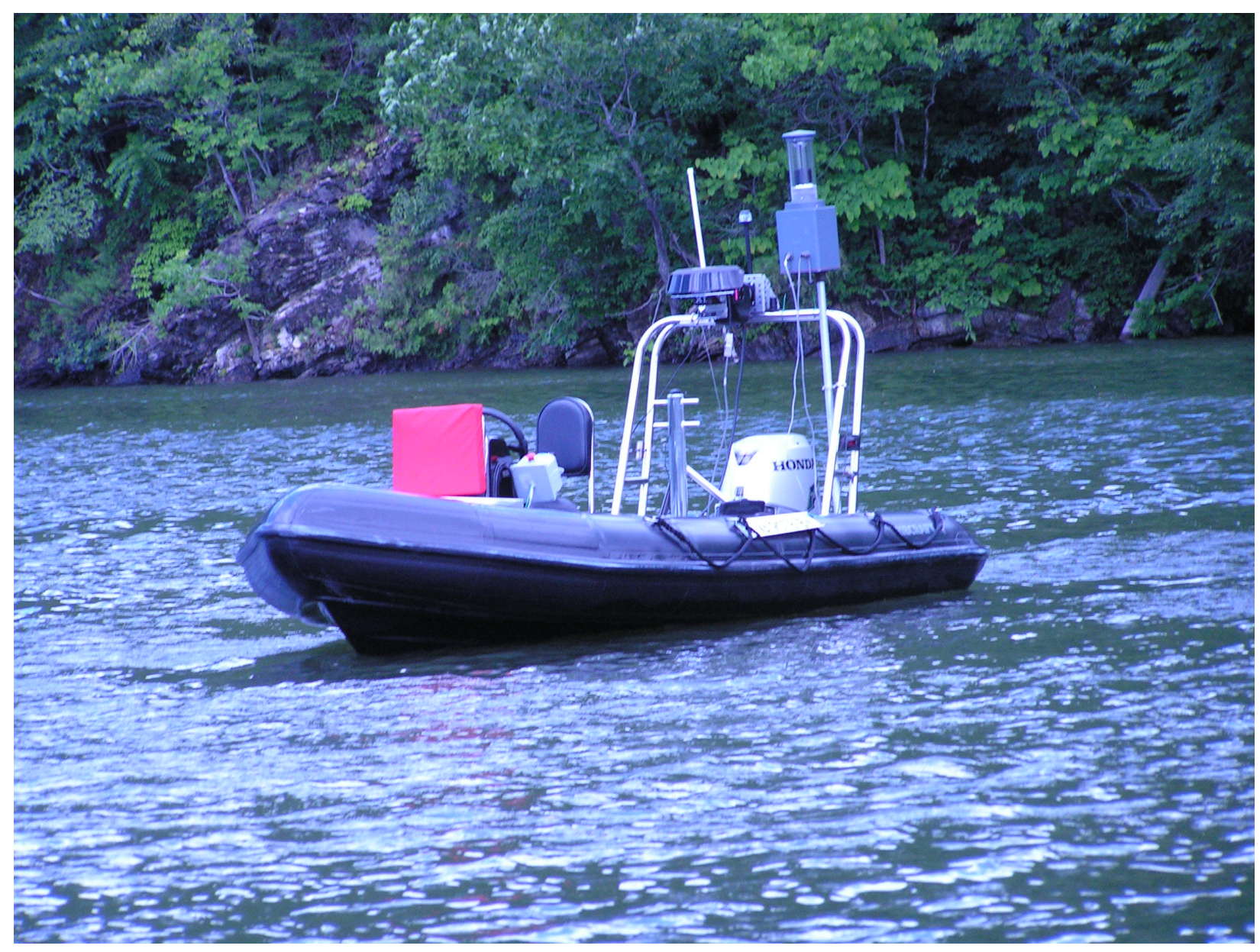

Figure 4: Ribcraft USV on Peak Creek

The SeaFox primary autopilot communicates with its electronic rudder and throttle control units over a controller area network (CAN), but also provides a serial communications interface for remote control operations through a radio modem. In this mode, an operator can program waypoints and monitor the vessel's mission status and real-time video via a shoreside operator control station. An operator may also take control of the vessel using a wearable joystick interface called the mobile remote operator station (MROS). The MROS allows direct control of the SeaFox rudder, throttle, and emergency stop functions. If necessary, SeaFox can also be driven manually.

In addition to these standard modes of operation, NPS has modified the USV to enable fully autonomous operations via an onboard PC/104 computer that acts as a secondary controller. This computer monitors onboard sensor data and sends overriding commands to the autopilot via the serial interface. During autonomous operations, MROS communications have highest priority and can interrupt commands from the secondary controller. This allows a human operator to take control at any time, thereby ensuring operational safety. Two-way command and control messages between SeaFox and the MROS or operator control station are sent via a Pacific Crest positioning data link (PDL) radio modem. In addition to this long-range, 9600-bps radio link, the NPS SeaFox has been modified to incorporate a Wave 


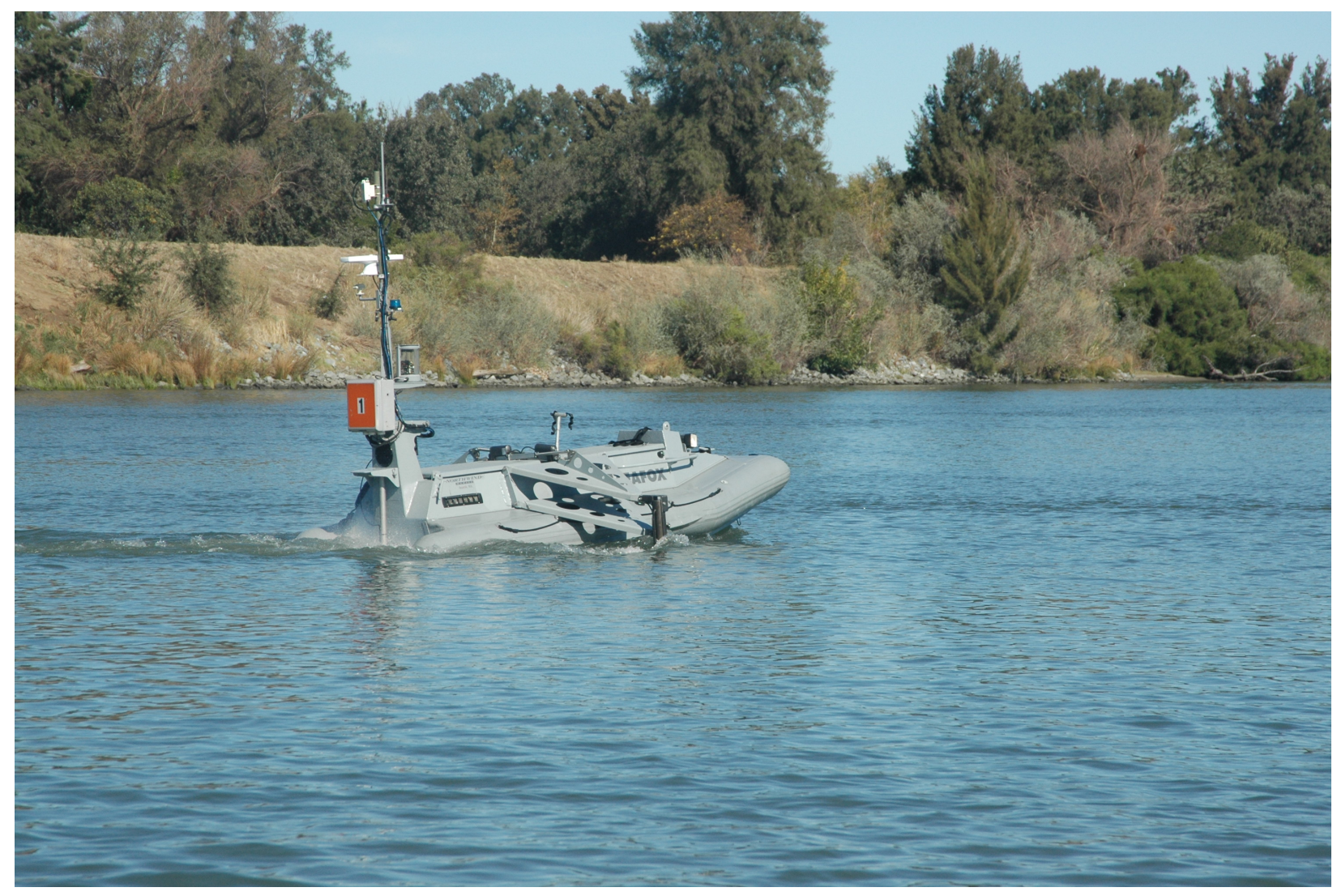

Figure 5: SeaFox operating on the Sacramento River

Relay mobile ad hoc network (MANET) from Persistent Systems. Wave Relay provides a scalable, high-performance wireless solution for collaborative networked operations and has been proven reliable when deployed in demanding maritime environments under the USSOCOM/NPS Field Experimentation Program. The 2.4GHz Wave Relay link allows operational commanders or other unmanned vehicles on a tactical network to receive realtime USV sensor data or send commands to the SeaFox secondary controller.

In autonomous mode, the NPS SeaFox navigates using a ComNav Vector G2 satellite compass to provide DGPS-based position updates and heading information. The USV has also been modified to include a Honeywell HG1700 inertial measurement unit (IMU) for precise attitude estimation. Standard sensors include fixed daylight and low-light navigation cameras (3 each) and dual Hood Technology AltiCam gyro-stabilized PTZ camera turrets (one daylight and one infrared version). All video is accessible over the network using two onboard Axis video servers. The AltiCams can be controlled via serial commands from the secondary controller, or by a networked operator using a joystick interface.

Since 2003, CAVR has been developing obstacle avoidance capabilities for autonomous underwater vehicles (AUVs) based on small, low power, forward-looking imaging sonar [?]. Leveraging this expertise to provide a subsurface sensing capability for USVs, NPS contracted with Northwind Marine for vehicle upgrades that allow SeaFox to deploy a BlueView sonar system below the waterline. The SeaFox sonar system is comprised of twin (port 
and starboard) $450-\mathrm{kHz}$ pole-mounted sonar heads attached to pan/tilt actuators. The entire system can be extended or retracted by remote control to allow low-speed sonar-based navigation in riverine environments or high-speed maneuvers in open water. Each blazed array sonar head provides a 45-degree by 15-degree field of view. The port and starboard sonar heads are mounted in a horizontal and vertical orientation, respectively, to produce two orthogonal image planes. These two perspectives provide different but complementary information about the subsurface environment to assist detection of obstacles in the waterway.

The NPS secondary controller is a 1.8-GHz PC/104 computer running the Mission Oriented Operating Suite (MOOS) under a Linux operating system. The MOOS architecture provides an open, client/server framework that facilitates interprocess communication between multiple software modules via a central database (the MOOSDB server). Each SeaFox task is implemented as a separate MOOS module that subscribes to the data it needs from other modules and publishes its output to the MOOSDB. In this manner, for example, the sonar pan/tilt module can respond to directives from the image-processing module to point at objects requiring further examination. Currently, all vehicle software is executed on a single computer, but one useful feature of the architecture is that MOOS clients can be distributed over multiple machines on a network. In the future, therefore, it will be possible to distribute the image-processing computational load across multiple processors as necessary.

\subsection{Pontoon USV}

Virginia Tech has also developed a small pontoon-style USV shown in Figure 6. One purpose for this vehicle is to investigate motion control algorithms for USV operations in significant currents, typically in the shallow waters of the New River, near Virginia Tech's main campus. The hull structure is a pair of inflatable kayaks fixed to an extruded aluminum frame. Two electric trolling motors are mounted at the midline, providing 40 pounds of thrust each and propelling the vehicle to a maximum speed of about 4 knots.

The Pontoon USV receives orientation information through an AHRS mounted within a dry-box. Location and velocity is determined using a Hemisphere DGPS. These instruments provide data at $10 \mathrm{~Hz}$ to an onboard computer located within the dry-box. The onboard computer also communicates with an on-shore base station. As a safety measure, the onboard laptop receives a heartbeat signal from the base station and times out if the heartbeat signal ceases for more than 2.5 seconds.

\section{System Identification Results}

Sea trials for the three vehicles are performed in order to implement the parameterization methods. The first task involves finding an open loop DC gain mapping of steady-state throttle and rudder setting to steady turn rate, forward velocity, and side velocity. Actuator dynamics are found by simply commanding extremal input commands and recording the

PAGE 17

Virginia Center for Autonomous Systems Report No. 2010-01 


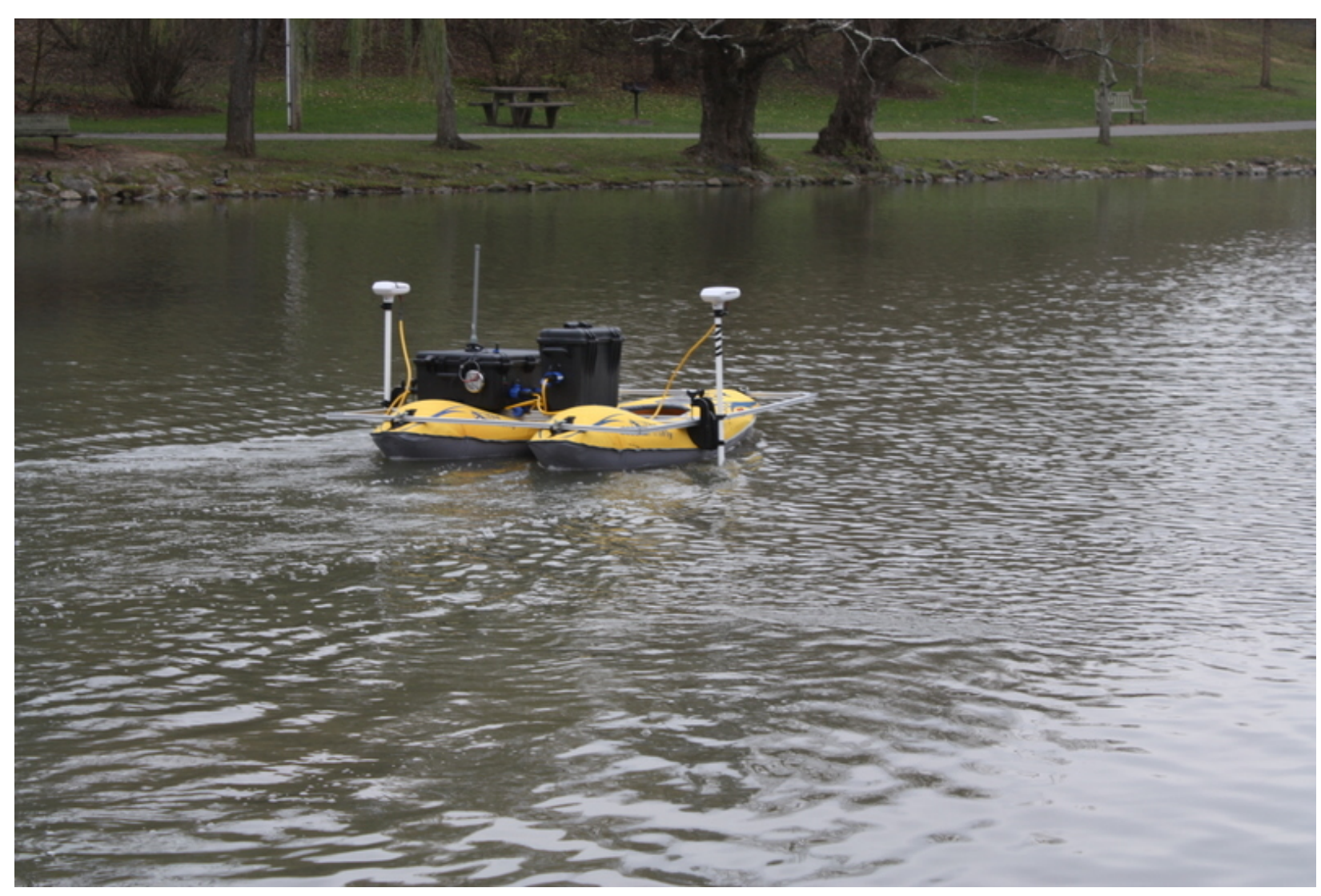

Figure 6: Pontoon USV operating in a pond on the VT campus

actual rudder and throttle settings. The closed loop field trials involve using square waves in commanded heading and velocity while recording time histories of the rudder, throttle, orientations, and velocities. The input/output and closed-loop simulation methodology use this closed loop field trial data. The input/output method neglects the commanded heading and velocity by looking into the interaction between rudder and throttle inputs to orientation and velocity outputs. The closed-loop simulation method uses all of the information available, which includes actuator dynamics and time histories of commanded heading and velocity.

\subsection{Steady-State Input/Output Parameters}

The detailed steps required to extracted open loop steady-state data are as follows:

1. Define $\tilde{\delta}=0^{\circ}$ and set the steering angle $\delta=\tilde{\delta}$ with the USV heading in a safe direction.

2. Increment throttle by 5\%. Allow speed to reach steady-state and take note of the planing status of the boat.

(a) Increment $\tilde{\delta}$ by a fixed amount (e.g., $5^{\circ}$ ). 
(b) Set $\delta=\tilde{\delta}$ and wait for the USV to complete two circular paths.

(c) Set $\delta=0$ and re-establish straight and steady motion.

(d) If $\tilde{\delta}$ is not at maximum, return to Step 2(a). Else, continue.

3. If the throttle is not at maximum, return to Step 2. Else, end the test sequence.

4. Perform steps 1 through 3 for negative (reverse) throttle settings.

These steps are performed for all three vehicles. Steady-state values for turn rate, sideslip angle, forward velocity, and side velocity are recorded for a range of constant throttle and rudder settings and presented for each boat. These steady-state values give rise to DC gains that are included in the parameterization procedure to ensure that the dynamic models act like the actual vehicle for steady-state maneuvers. The gains are computed uniquely for each model based upon the corresponding assumptions. For instance if a small rudder angle is assumed such that $\sin \delta \approx \delta$, implying linear input, only the steady-state values closest to zero rudder input will be used. The gain mapping is a curve fit for nonlinear inputs. For the decoupled steering and throttle models these gain maps will only be based upon either steering or throttle, but not both. For the full nonlinear planar model, which does not decouple steering and throttle, a three dimensional curve fit including both steering and throttle is required.

The Ribcraft USV steady-state results can be seen in table 1 and figure 7 . The Ribcraft USV has a dead band in throttle from 0 to $30 \%$ and the throttle input ranges from $20 \%$ to $65 \%$ throttle. The rudder input ranges from 0 to $15^{\circ}$ rudder angle, which produces only right hand turns. The turn rate can be segregated into a low speed and high speed region with turn rate increasing with throttle setting and rudder angle. Forward velocity increases with throttle but decreases with rudder angle since energy is being expended to give a centripetal turning force as well as the vectored thrust being used to induce a moment. Sideslip angle increases with throttle and rudder angle. Assuming the boat acts like a hovercraft at slow speed would be considered a valid assumption, but the data does not reflect this. The rudder that is part of the linkage to the propellor keeps the boat stable at slow speeds, which keeps the angle of sideslip from becoming large. There is a "sweet spot" for high sideslip angles in the speed region right before the transition to planing travel. At this speed region the boat does not lean into turns, which would provide greater drag in side to side translation. The large sideslip angle outlier at the highest speed is due to water cavitation in front of the propellor causing a severe loss of thrust.

The Seafox USV steady-state results can be seen in table 2 and figure 8. Data taken for the Seafox USV includes increments of $5^{\circ}$ steering angle in the appendix. The Seafox USV's entire steady-state collection is roughly in its dead band zone since the forward velocity, sideslip angle, and turn rate have no appreciable change based on throttle setting. Again, only right hand turns are considered, which range from $0^{\circ}$ to $30^{\circ}$ rudder angle. This jet boat does indeed act like a hovercraft at low speeds since the vectored thrust is implemented with no effect of rudder. This can be seen in the exceptionally high sideslip angles at maximum rudder angle. The turn rate and sideslip angle increase with rudder angle, while the forward velocity decreases.

PAGe 19

Virginia Center for Autonomous Systems Report No. 2010-01 
Table 1: Steady-state values of $u, \beta$, and $r$ for the Ribcraft USV.

\begin{tabular}{|c|c|c|c|c|c|c|c|c|c|c|c|}
\hline \multirow{2}{*}{\multicolumn{2}{|c|}{ Steering angle, $\delta r$}} & \multicolumn{4}{|c|}{ Forward speed, $u(\mathrm{~m} / s)$} & \multicolumn{3}{|c|}{ Sideslip angle, $\beta$ (deg) } & \multicolumn{3}{|c|}{ Turn rate, $r(\operatorname{deg} / s)$} \\
\hline & & $0^{\circ}$ & $5^{\circ}$ & $10^{\circ}$ & $15^{\circ}$ & $5^{\circ}$ & $10^{\circ}$ & $15^{\circ}$ & $5^{\circ}$ & $10^{\circ}$ & $15^{\circ}$ \\
\hline \multirow{9}{*}{ Throttle, $\delta T$} & $20 \%$ & 1.1 & 2.0 & 1.8 & 1.7 & -10.1 & -23.7 & -25.7 & 3.0 & 5.6 & 7.5 \\
\hline & $30 \%$ & 1.1 & 2.0 & 1.8 & 1.7 & -14.7 & -17.7 & -25.1 & 3.2 & 5.7 & 8.2 \\
\hline & $35 \%$ & 1.5 & 2.8 & 2.5 & 2.1 & -11.8 & -21.2 & -37.1 & 4.0 & 7.7 & 10.8 \\
\hline & $40 \%$ & 2.2 & 3.9 & 3.4 & 2.5 & -15.7 & -28.2 & -48.2 & 6.8 & 10.4 & 13.5 \\
\hline & $45 \%$ & 2.7 & 5.1 & 4.7 & 3.7 & -8.9 & -22.3 & -42.1 & 7.0 & 11.3 & 15.7 \\
\hline & $50 \%$ & 3.0 & 5.8 & 5.5 & 4.3 & -6.1 & -15.6 & -40.5 & 7.0 & 14.0 & 22.4 \\
\hline & $55 \%$ & 3.6 & 6.9 & 6.8 & 5.7 & -14.1 & -11.1 & -32.4 & 7.4 & 14.8 & 22.3 \\
\hline & $60 \%$ & 4.0 & 7.0 & 6.9 & 6.6 & -13.4 & -13.0 & -17.4 & 6.6 & 13.6 & 22.0 \\
\hline & $65 \%$ & 5.1 & 9.9 & 8.0 & 6.0 & -9.3 & -34.8 & -50.6 & 7.2 & 13.8 & 21.8 \\
\hline
\end{tabular}

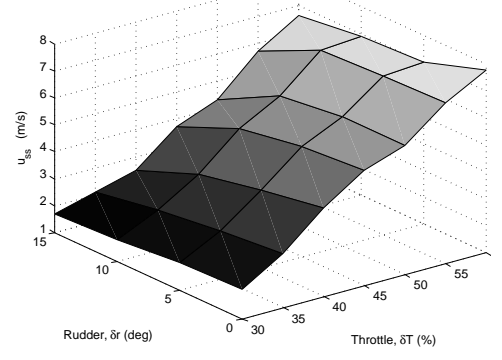

(a) Forward Velocity

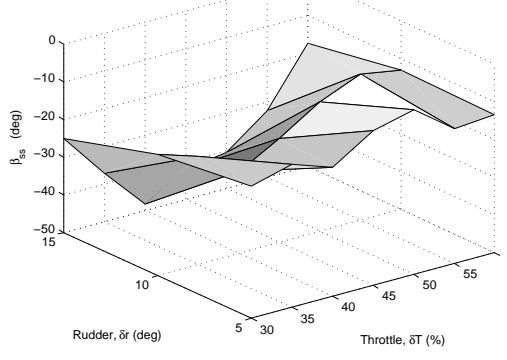

(b) Sideslip Angle

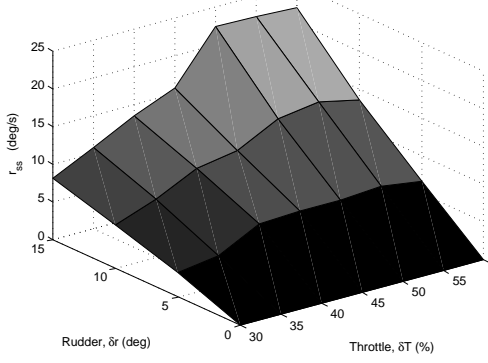

(c) Turn Rate

Figure 7: Ribcraft USV steady-state data.

Table 2: Steady-state values of $u, \beta$, and $r$ for the Seafox USV.

\begin{tabular}{|c|c|c|c|c|c|c|c|c|c|c|c|}
\hline & & \multicolumn{4}{|c|}{ Forward speed, $u(\mathrm{~m} / s)$} & \multicolumn{3}{|c|}{ Sideslip angle, $\beta$ (deg) } & \multicolumn{3}{|c|}{ Turn rate, $r(\operatorname{deg} / s)$} \\
\hline Steering ang & $\delta r$ & $0^{\circ}$ & $10^{\circ}$ & $20^{\circ}$ & $30^{\circ}$ & $10^{\circ}$ & $20^{\circ}$ & $30^{\circ}$ & $10^{\circ}$ & $20^{\circ}$ & $30^{\circ}$ \\
\hline \multirow{5}{*}{ Throttle, $\delta T$} & $15 \%$ & 0.9 & 0.9 & 0.7 & 0.2 & -22.6 & -41.7 & -75.6 & 5.7 & 8.9 & 14.1 \\
\hline & $20 \%$ & 0.4 & 1.0 & 0.6 & 0.1 & -21.6 & -48.4 & -83.5 & 5.1 & 9.5 & 13.5 \\
\hline & $25 \%$ & 1.5 & 1.1 & 0.5 & 0.0 & -20.8 & -56.7 & -89.0 & 5.2 & 11.4 & 16.5 \\
\hline & $30 \%$ & 1.2 & 1.8 & 1.5 & 0.6 & -18.4 & -33.4 & -65.8 & 7.9 & 12.6 & 20.7 \\
\hline & $35 \%$ & 0.9 & 2.1 & 1.5 & 0.6 & -17.8 & -39.4 & -69.4 & 8.3 & 16.1 & 23.4 \\
\hline
\end{tabular}

The Pontoon USV steady-state results can be seen in table 3 and figure 9. There is no dead band zone for the Pontoon USV's electric trolling motors. This being the case, forward velocity increases with every increase in throttle. Consider only right hand turns, which relates to positive throttle mixing. After $25 \%$ steering mixing the forward velocity and 


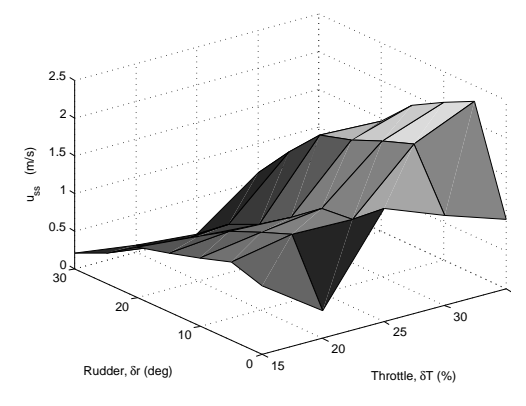

(a) Forward Velocity

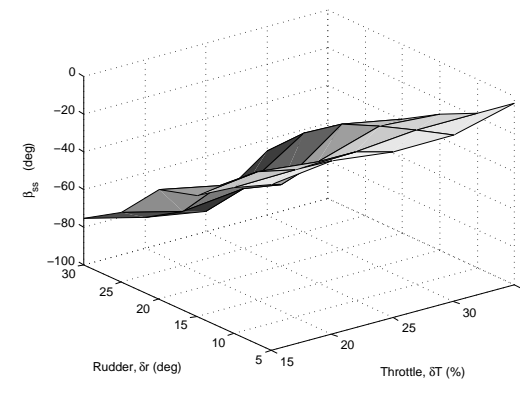

(b) Sideslip Angle

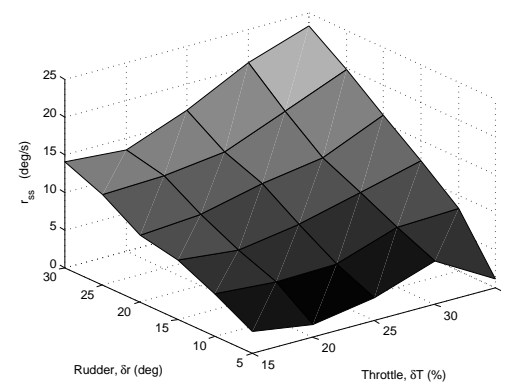

(c) Turn Rate

Figure 8: Seafox USV steady-state data.

sideslip angle become uniform across all throttle settings. The sideslip angle is moderate when a forward velocity is maintained in the turn. When the forward velocity deteriorates to almost zero the side slip angle hovers around $90^{\circ}$. Turn rate is not dependent upon forward speed. For all throttle settings the turn rate is uniform and increases linearly with steering mixing.

Table 3: Steady-state values of $u, \beta$, and $r$ for the Pontoon USV.

\begin{tabular}{r||ccccc|ccccc|cccc}
\multicolumn{1}{l||}{} & \multicolumn{4}{c|}{ Forward speed, $u(\mathrm{~m} / s)$} & \multicolumn{4}{c|}{ Sideslip angle, $\beta(\mathrm{deg})$} & \multicolumn{4}{c}{ Turn rate, $r(\mathrm{deg} / s)$} \\
\hline \multicolumn{1}{c||}{ Steering mixing, $\%$} & $0 \%$ & $25 \%$ & $50 \%$ & $75 \%$ & $100 \%$ & $25 \%$ & $50 \%$ & $75 \%$ & $100 \%$ & $25 \%$ & $50 \%$ & $75 \%$ & $100 \%$ \\
\hline \hline \multirow{2}{*}{ Throttle, $\delta T$} & 0.3 & 0.0 & -0.0 & -0.0 & 0.0 & -87.0 & -104.5 & -106.2 & -97.6 & 21.4 & 32.3 & 44.0 & 53.5 \\
& $40 \%$ & 0.8 & 0.1 & -0.1 & -0.1 & -0.1 & -77.4 & -105.7 & -113.4 & -108.4 & 25.4 & 39.9 & 47.7 & 56.6 \\
& $60 \%$ & 1.2 & 0.5 & -0.1 & -0.1 & -0.3 & -38.1 & -100.6 & -100.1 & -120.7 & 21.1 & 45.8 & 53.3 & 60.2 \\
$80 \%$ & 1.5 & 0.8 & 0.2 & 0.2 & -0.3 & -32.7 & -68.0 & -70.5 & -121.5 & 15.1 & 42.8 & 60.0 & 64.0 \\
& $100 \%$ & 1.8 & 1.0 & 0.3 & 0.1 & 0.2 & -30.7 & -70.3 & -84.4 & -80.7 & 13.5 & 38.4 & 57.9 & 69.4 \\
\hline
\end{tabular}

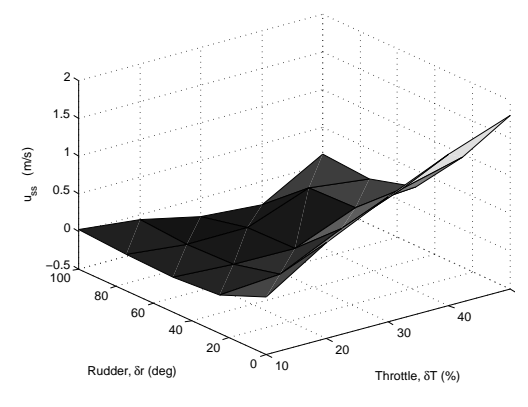

(a) Forward Velocity

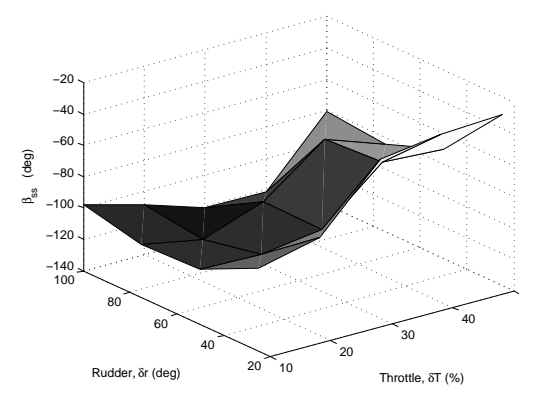

(b) Sideslip Angle

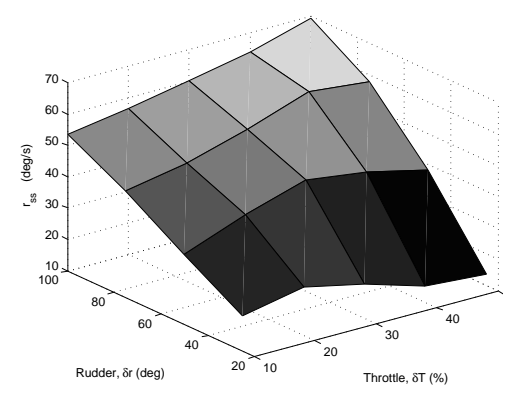

(c) Turn Rate

Figure 9: Pontoon USV steady-state data.

DC Gains. In Section 3.1, the method to obtain the "DC gains" which enforce steadystate motion for the linear models, is discussed. Using data from Section 5.1 the Ribcraft 
USV, Seafox USV, and Pontoon USV "DC gains" are shown in tables 4, 5, and 6. Note the definition of "DC gain" in this report is $K_{*}=\frac{*_{\mathrm{ss}}}{\delta}$ where $\delta$ can be either throttle or steering angle.

Table 4: Steady-state DC gains of $u, \beta, v$, and $r$ for the Ribcraft USV.

\begin{tabular}{c||cccc} 
Throttle, $\delta T$ & $K_{u}$ & $K_{\beta}$ & $K_{v}$ & $K_{r}$ \\
\hline \hline $30 \%$ & -0.13 & -1.9 & -0.063 & 0.52 \\
$35 \%$ & 0.14 & -1.8 & -0.059 & 0.56 \\
$40 \%$ & 0.22 & -2.4 & -0.11 & 0.74 \\
$45 \%$ & 0.23 & -3.1 & -0.19 & 0.97 \\
$50 \%$ & 0.15 & -2.6 & -0.21 & 1.1 \\
$55 \%$ & 0.17 & -2.3 & -0.21 & 1.5 \\
$60 \%$ & 0.20 & -1.9 & -0.22 & 1.5 \\
$65 \%$ & 0.29 & -1.3 & -0.16 & 1.4 \\
\hline
\end{tabular}

Table 5: Steady-state DC gains of $\beta, v$, and $r$ for the Seafox USV.

\begin{tabular}{c||ccc} 
Throttle, $\delta T$ & $K_{\beta}$ & $K_{v}$ & $K_{r}$ \\
\hline \hline $15 \%$ & -2.41 & -0.015 & 0.48 \\
$20 \%$ & -2.63 & -0.013 & 0.47 \\
$25 \%$ & -2.77 & -0.013 & 0.55 \\
$30 \%$ & -1.95 & -0.031 & 0.68 \\
$35 \%$ & -2.11 & -0.033 & 0.79 \\
\hline
\end{tabular}

Table 6: Steady-state DC gains of $\beta, v$, and $r$ for the Pontoon USV.

\begin{tabular}{c||ccc} 
Throttle, $\delta T$ & $K_{\beta}$ & $K_{v}$ & $K_{r}$ \\
\hline \hline $10 \%$ & -1.42 & -0.0048 & 0.63 \\
$20 \%$ & -1.36 & -0.0053 & 0.68 \\
$30 \%$ & -1.15 & -0.0053 & 0.72 \\
$40 \%$ & -1.00 & -0.0077 & 0.72 \\
$50 \%$ & -2.11 & -0.033 & 0.79 \\
\hline
\end{tabular}

Steady-State Mapping. In Section 3.1, the method to obtain the throttle and steering angle steady-state mapping, which enforce steady-state motion for the nonlinear models, is discussed. Using data from Section 5.1 the Ribcraft USV steady-state mapping coefficients are shown as

$$
\boldsymbol{K}=\left(\begin{array}{ccc}
-3.8 & 0.20 & -0.0023 \\
0 & 0 & -0.0036 \\
0 & 0 & 0.025
\end{array}\right)
$$

in (28). Notice that there is a non-zero bias in $u_{\mathrm{SS}}$, which dictates a non-zero forward velocity for a zero throttle setting. The throttle to forward velocity mapping does not describe the nonlinear actuator dynamics, which occur for throttle settings below $30 \%$. 


\subsection{Actuator Dynamics}

The actuators affect the dynamics by augmenting the potentially infeasible commanded outputs. The Ribcraft USV has saturation limited rudder input due to the steering assembly and mounting to the boat, giving a maximum of $\pm 20^{\circ}$ of rudder. The rudder is rate limited due to the limitation on the hydraulic motor feeding into the steering assembly. The nature of this hydraulic assembly is that of high torque but low speed. The rudder very quickly reaches a maximum slew rate and stays there as long as required regardless of the water forces experienced by the boat. The rate limiting is $\pm 5.6^{\circ} / \mathrm{s}$. The throttle is considered to give near instantaneous manifold pressure to the engine. The dynamics of the engine and propellor give the desired thrust but are deemed to complex to be determined without further separate testing of each component. What can be measured is a dead band in the throttle for $\pm 30 \%$ when the engine and propellor is engaged, which gives a constant thrust. When the throttle is at $0 \%$ the engine disengages from the rudder providing no thrust.

The Seafox USV has a saturation limited steering angle of $\pm 30^{\circ}$ due to mechanics in the thrust vectoring assembly. Actual steering angle is not measured, so no assessment of the dynamics of the actuator can be done. Since only a nozzle is moved it is assumed that the actuator has fast dynamics that can be ignored. Again, the throttle is considered to give near instantaneous manifold pressure to the engine. The dead zone of the throttle is at least as high or higher than that of the Ribcraft USV. Since the closed loop data sets do not exceed 30\% throttle, the Seafox USV's throttle dynamics are not considered.

Only throttle actuator assessment is needed for the Pontoon USV. The electric motors are considered to receive near instantaneous voltage giving no throttle dynamics. The maximum throttle is limited to $50 \%$ and the steering mixing is $\pm 100 \%$ which correlates to a difference of 6 volts.

\subsection{Dynamics from Input/Output Data}

Figures 10 and 11 show representative time histories (actual and simulated) for the feedback controlled Ribcraft USV in response to commanded square waves in speed and course angle, respectively. In Figure 10, it is clear that the first order speed model identified for the given throttle setting fails to capture the detailed behavior of the vehicle in surge. While the steady-state behavior of the linear model matches that of the true system, by construction, only the gross trend in the dynamic response is captured by the linear model.

For the data shown in Figure 11, it appears that the Nomoto model with sideslip and linear model best approximates the true course angle history, an observation that is supported by comparative analysis of the parameter identification cost function data. It is somewhat surprising that the Nomoto model with sideslip should be equal to or outperform the linear model, which contains two more parameters (representing zeros in the transfer functions from steering angle to sideslip angle and turn rate, respectively). This discrepancy is rectified when one realizes the cost function values and time history plots were computed using sets of data independent of the data used for model identification. The linear model with two

PAGe 23

Virginia Center for Autonomous Systems Report No. 2010-01 


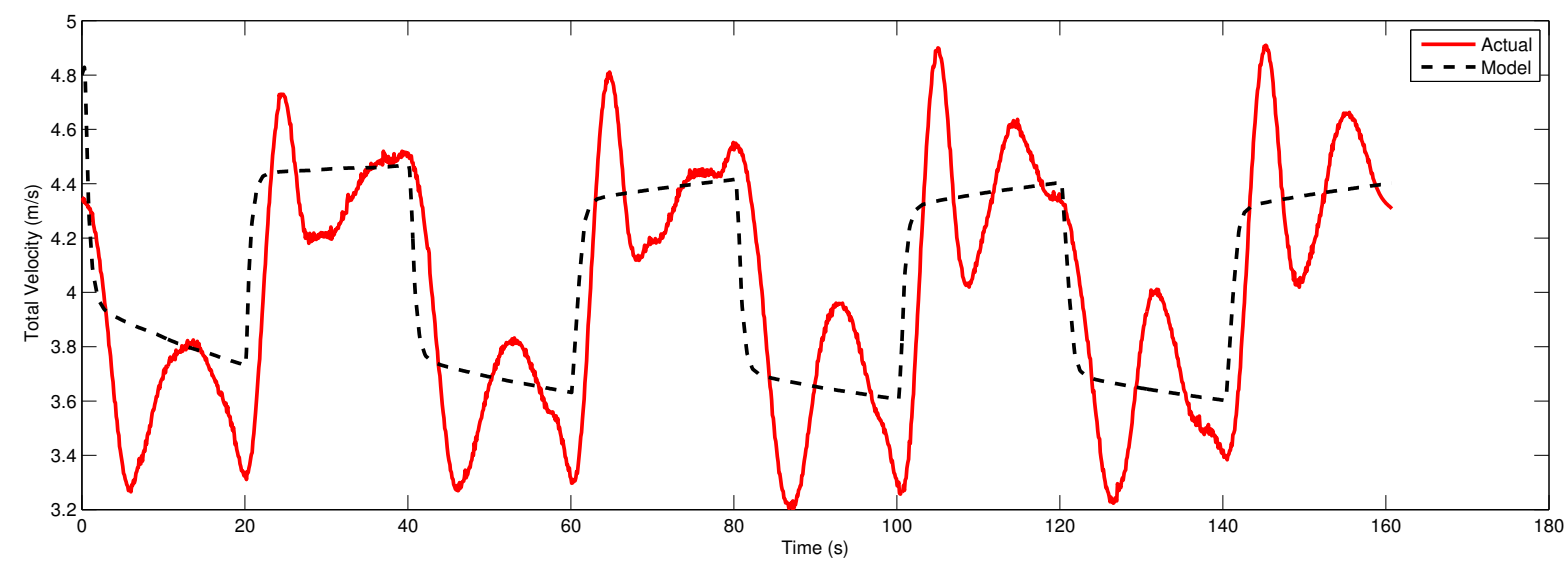

Figure 10: Time histories for speed (actual and simulated) for the Ribcraft in straight-line motion.

more parameters will be able to better model a particular set of data, but not be general enough for independent sets of data. The Nomoto model with sideslip provides a reasonable representation of the steering dynamics at a given throttle setting.

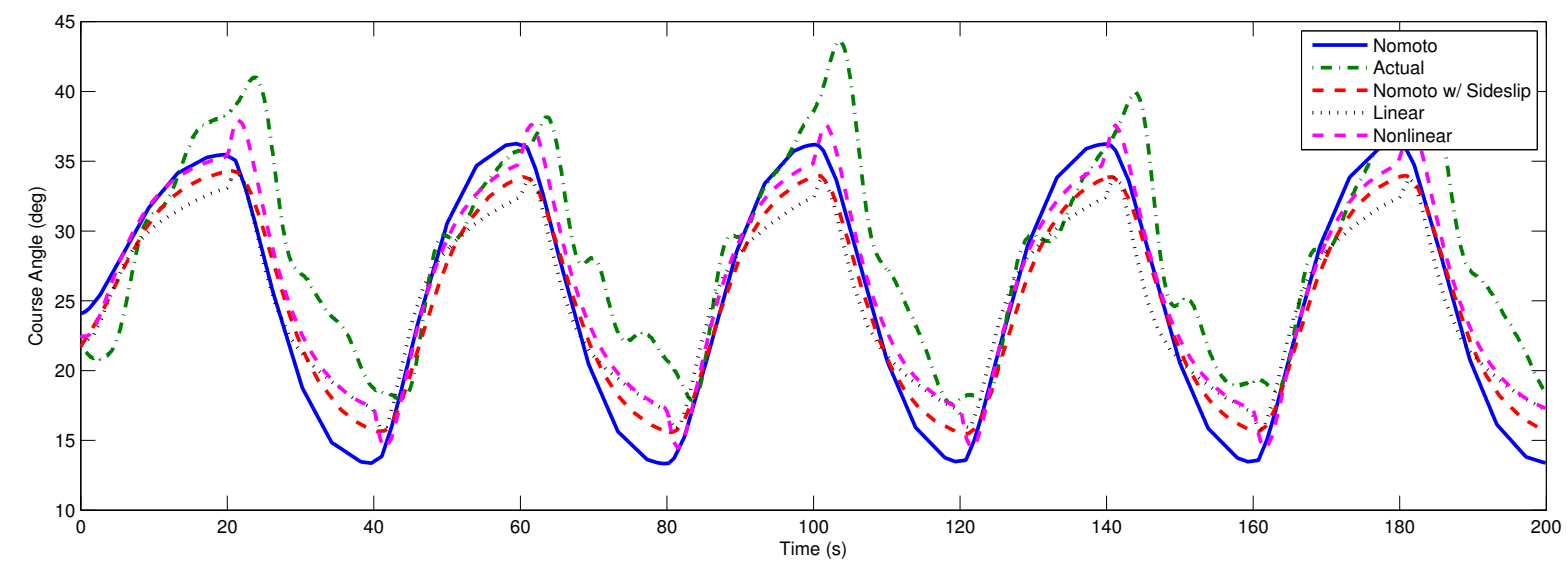

Figure 11: Representative time histories of course angle (actual and simulated) for the Ribcraft USV in straight-line motion.

Figure 12 shows course angle data for the Pontoon USV accompanied by the three identified linear steering models. The Nomoto model with sideslip also performs the best at approximating the true course angle history for all throttle settings. 


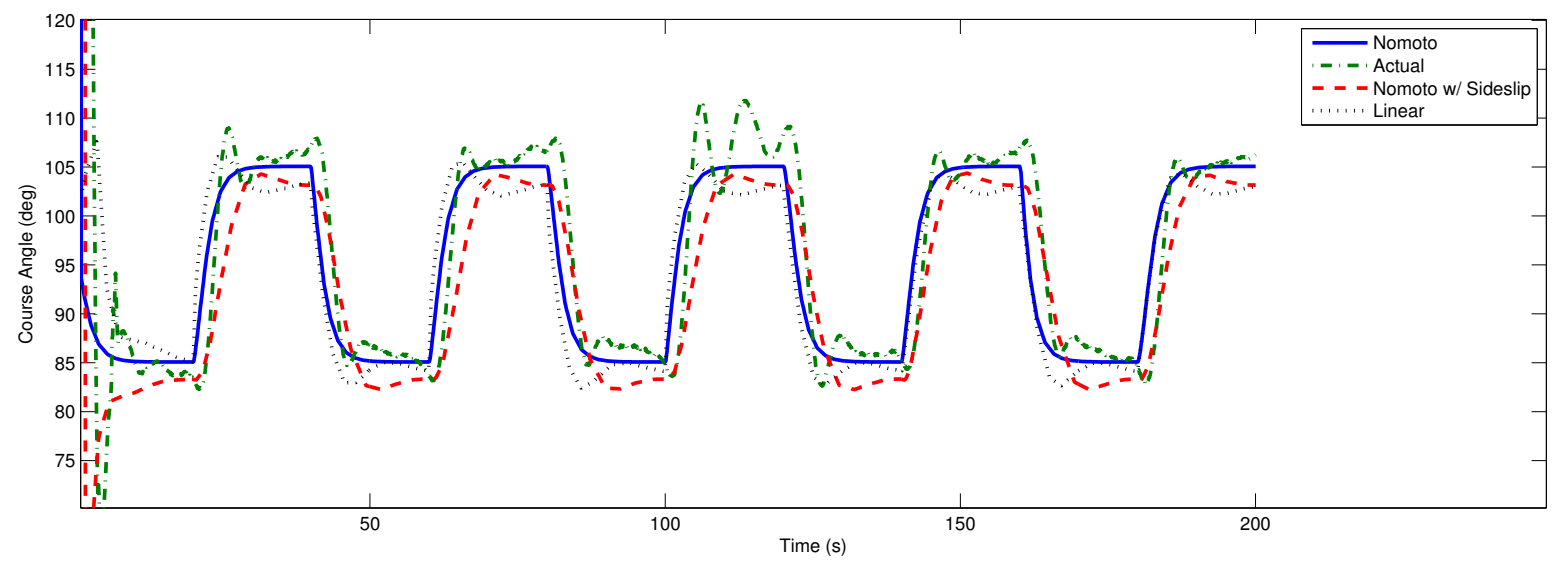

Figure 12: Time histories of course angle (actual and simulated) for the Pontoon USV in straight-line motion.

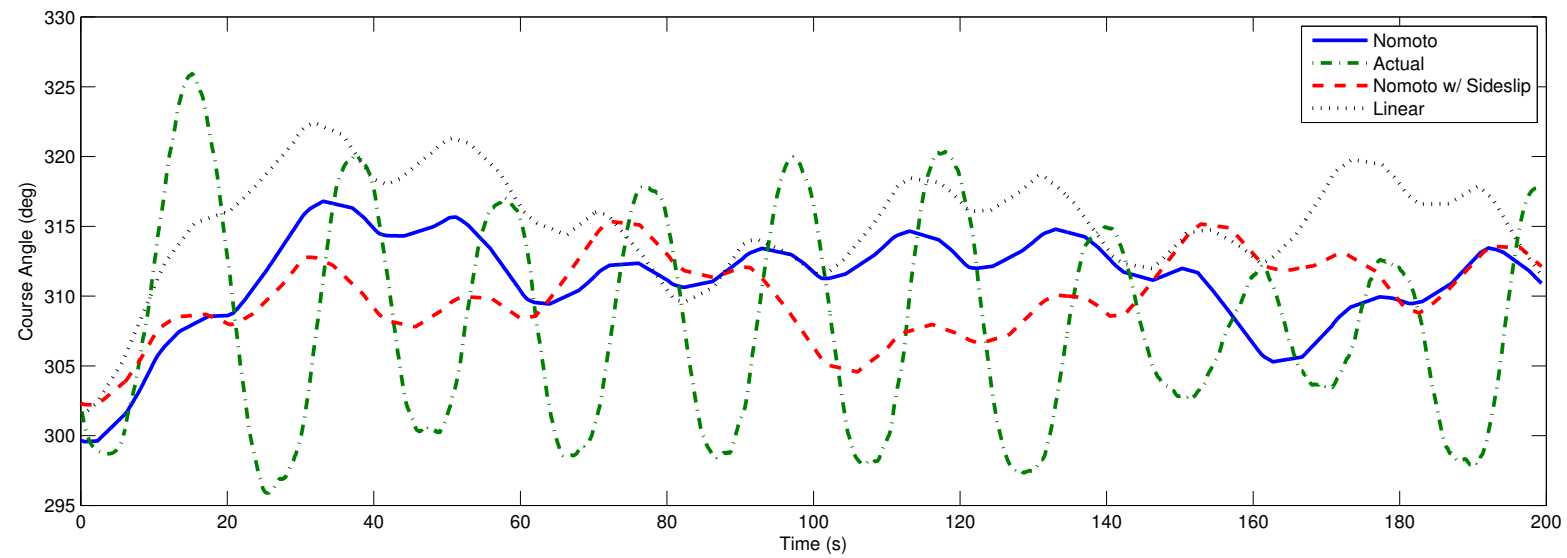

Figure 13: Time histories of course angle (actual and simulated) for the Seafox USV in straight-line motion.

For the data shown in Figure 13, none of the models have a clear advantage of the other. In fact, the models do not accurately represent the dynamics of the Seafox USV. The poor quality of the model matching is due to disparity between the steady-state and closed loop field trials. The steady-state turn rates defined by constant turning maneuvers are too small when used with the closed loop field trials. 
Table 7: Steering cost function values for the Ribcraft USV.

\begin{tabular}{c||cccc} 
Throttle, $\delta T$ & Nomoto & Nomoto w/ Sideslip & Linear & Nonlinear \\
\hline \hline $20 \%$ & 0.37 & 0.37 & 0.54 & 23.51 \\
$30 \%$ & 0.45 & 0.39 & 0.59 & 0.65 \\
$35 \%$ & 0.45 & 0.33 & 0.48 & 0.51 \\
$40 \%$ & 0.31 & 0.46 & 0.37 & 0.66 \\
$45 \%$ & 0.42 & 0.37 & 0.37 & 0.53 \\
$50 \%$ & 0.26 & 0.33 & 0.30 & 0.50 \\
$55 \%$ & 0.24 & 0.49 & 0.24 & 0.72 \\
$60 \%$ & 0.42 & 0.39 & 0.38 & 0.26 \\
$65 \%$ & 0.27 & 0.39 & 0.34 & 1.78 \\
\hline
\end{tabular}

Table 7 shows cost function values for identified models of the Ribcraft USV steering dynamics. These values were computed using data sets that are independent of the data sets used for model identification. In each case the Nomoto model with sideslip outperforms the other models. The superiority of the Nomoto model with sideslip can be attributed to its lack of exhaustive linear parameters while still properly capturing the essences of the dynamics. The linear steering modeling may find a very accurate model for a given data set that is not general enough for all data sets at that particular throttle setting.

Table 8: Speed cost function values for the Ribcraft USV.

\begin{tabular}{c||cc} 
Speed Region, m/s & Linear & Nonlinear \\
\hline \hline 1.0 & 1.35 & 3.90 \\
1.5 & 2.83 & 2.91 \\
2.0 & 2.27 & 3.14 \\
2.5 & 1.11 & 3.22 \\
3.0 & 1.14 & 2.10 \\
3.5 & 1.61 & 2.37 \\
4.0 & 2.20 & 2.91 \\
\hline
\end{tabular}

Table 9 shows cost function values for the Pontoon USV showing the Nomoto model with sideslip performing the best.

Table 9: Steering cost function values for the Pontoon USV.

\begin{tabular}{c||ccc} 
Throttle, $\delta T$ & Nomoto & Nomoto w/ Sideslip & Linear \\
\hline \hline $40 \%$ & 1.07 & 0.822 & 1.41 \\
$50 \%$ & 1.28 & 1.12 & 1.26 \\
\hline
\end{tabular}

Table 10 shows cost fucntion values for the Seafox USV showing the Nomoto model and Nomoto model with sideslip performing the best. Best is a relative term and does not imply 
that these values are practical for use, due to the disparity between steady-state and realized closed loop input relationships.

Table 10: Steering cost function values for the Seafox USV.

\begin{tabular}{c||ccc} 
Throttle, $\delta T$ & Nomoto & Nomoto w/ Sideslip & Linear \\
\hline \hline $15 \%$ & 0.724 & 0.790 & 0.933 \\
$20 \%$ & 0.710 & 0.660 & 0.854 \\
$25 \%$ & 0.742 & 0.653 & 0.663 \\
\hline
\end{tabular}

Lag between the sensors and the computer logging the data can have a large effect upon the end results. This lag manifests itself most significantly in initializing step in the parameterization method. The affected initialization skews the end results due to local minima existing in the search space of the optimization. For instance, if a system is defined unstable in the initializing step, the closed loop parameterization will find the best unstable parameter set. Other local minima may exist for stable models as well. The initialization step also requires higher order derivatives than the final parameterization, which adds greater numeric uncertainty.

\section{Conclusion}

Results of experimental motion data analysis were presented for three USVs, along with a comparison of simple dynamic models identified using the data. Each platform represents a distinct control system implementation: an outboard engine, a jet-drive thruster, and differential electric thrusters. In each case, the USVs were observed to experience significant sideslip. For vehicles moving fast enough that GPS provides reliable course angle data, the steering dynamics can be well-approximated by first order lag models for turn rate and sideslip, a four-parameter steering model, and a 16 parameter coupled nonlinear model can be easily identified from motion data. The linear models provide the best fit to actual time histories but require parameters to change based upon the nominal throttle setting. The nonlinear coupled model fits the actual time histories adequately and has constant parameters through the entire maneuvering envelope. 


\section{References}

[1] A. S. Aditya Gadre, S. Kragelund, T. Masek, D. Stilwell, C. Woolsey, and D. Horner. Subsurface and surface sensing for autonomous navigation in a riverine environment. In In Proceedings to AUVSI's Unmanned Systems, Washington, D. C., 2009.

[2] Volker Bertram. Unmanned surface vehicles - a survey.

[3] M. Breivik, V. E. Hovstein, and T. I. Fossen. Straight-line target tracking for unmanned surface vehicles. Modeling, Identification, and Control, 29(4):131-149, 2008.

[4] O. M. Faltinsen. Hydrodynamics of High-Speed Marine Vehicles. Cambridge University Press, 2005.

[5] T. I. Fossen. Guidance and Control of Ocean Vehicles. John Wiley and Sons, 1995.

[6] M. S. Grewal and K. Glover. Identifiability of linear and nonlinear dynamical systems. In IEEE Transaction on Automatic Control, December 1976.

[7] H. Lamb. Hydrodynamics. Dover, New York, sixth edition, 1932.

[8] PEO LMW. The navy unmanned surface vehicle (usv) master plan. 2007.

[9] J.E. Manley. New tools for ocean exploration, equipping the noaa ship okeanos explorer. In OCEANS 2008, Quebec City, Quebec, Canada, 2008.

[10] K. Nomoto, T. Taguchi, K. Honda, and S. Hirano. On the steering qualities of ships. Technical report, International Shipbuilding Progress, Vol. 4, 1957.

[11] Jan Petrich. Improved Guidance, Navigation, and Control for Autonomous Underwater Vehicles: Theory and Experiment. PhD thesis, Virginia Polytechnic Institute and State University, Blacksburg, Virginia, 2009.

[12] C. Sonnenburg, A. Gadre, D. Horner, S. Kragelund, D. J. Stilwell, and C. A. Woolsey. Control-oriented planar motion modeling of unmanned surface vehicles. In OCEANS 2010, Seattle, WA, 2010.

[13] R. F. Stengel. Optimal Control and Estimation. Dover Publications, Mineola, NY, 1986.

[14] Z. Yu, X. Bao, and K. Nonami. Course keeping control of an autonomous boat using low cost sensors. Journal of System Design and Dynamics, 2(1):389-400, November 2008. 


\section{Appendices}

\section{A Ribcraft USV Parameters and Time Histories}

Note that $a_{*}$ and $b_{*}$ refer to the elements $*$ in the state matrix $\boldsymbol{A}$ and input matrix $\boldsymbol{B}$ respectively. If $a$ and $b$ appear without a subscript, then the state and input matrices are scalars.

\section{A.1 Parameters}

Table 11: Ribcraft USV nonlinear (??) parameter values (using linearly parameterized fit of steady-state gains). All values of $\boldsymbol{A}$ that aren't shown in the table are implied to be zero.

\begin{tabular}{c|c||c|c}
\multicolumn{2}{c}{ state parameters } & \multicolumn{2}{c}{ input parameters } \\
\hline \hline$a_{11}$ & -0.22 & $b_{1_{1}}$ & -0.43 \\
$a_{14}$ & 0.0013 & $b_{1_{\delta T}}$ & 0.023 \\
$a_{22}$ & -0.74 & $b_{1_{\delta T|\delta r|}}$ & -0.00026 \\
$a_{25}$ & -0.027 & $b_{1_{\delta T^{2} \delta r^{2}}}$ & -0.00000012 \\
$a_{33}$ & -0.89 & $b_{2_{\delta T \delta r}}$ & -0.0040 \\
$a_{36}$ & 0.30 & $b_{2_{\delta T^{2} \delta r}}$ & 0.000071 \\
& & $b_{2_{\delta T^{2}|\delta r| \delta r}}$ & -0.00000080 \\
& & $b_{3_{\delta T \delta r}}$ & 0.020 \\
& & $b_{3_{\delta T^{2} \delta r}}$ & 0.00011 \\
& & $b_{3_{\delta T^{2}|\delta r| \delta r}}$ & -0.0000013 \\
\hline
\end{tabular}

Table 12: Ribcraft USV linear (21) parameter values (using linear fit of steady-state gains).

\begin{tabular}{rc||cccccc}
\multicolumn{2}{c}{ parameters } & $a_{11}$ & $a_{12}$ & $a_{21}$ & $a_{22}$ & $b_{1}$ & $b_{2}$ \\
\hline \hline $20 \%$ & -0.040 & -0.0072 & 2.19 & -0.091 & 0.0012 & 0.19 \\
$30 \%$ & -0.0048 & 0.0058 & 0.66 & -0.19 & -0.0035 & 0.15 \\
$35 \%$ & -0.017 & -0.00031 & -0.64 & -1.18 & -0.0016 & 0.80 \\
throttle (\%) & $45 \%$ & -0.012 & -0.015 & 1.27 & -0.31 & 0.013 & 0.54 \\
& $40 \%$ & -0.29 & -0.029 & 3.17 & -0.15 & -0.029 & 0.82 \\
& $50 \%$ & -0.28 & -0.030 & 3.03 & -0.15 & -0.016 & 0.85 \\
$55 \%$ & -0.22 & -0.031 & 2.15 & -0.063 & -0.0020 & 0.56 \\
$60 \%$ & -0.14 & -0.015 & 1.57 & -0.037 & -0.00095 & 0.30 \\
$65 \%$ & 0.0096 & -0.012 & 0.18 & -0.21 & 0.022 & 0.38
\end{tabular}

\section{A.2 Time Histories}


Table 13: Ribcraft USV Nomoto-with-sideslip (25) parameter values (using linear fit of steady-state gains).

\begin{tabular}{cc||cccc}
\multicolumn{2}{c}{ parameters } & $a_{11}$ & $a_{13}$ & $a_{33}$ & $b_{3}$ \\
\hline \hline & $20 \%$ & -0.021 & -0.079 & -0.28 & 0.15 \\
& $30 \%$ & -0.28 & -0.89 & -0.25 & 0.14 \\
& $35 \%$ & -0.35 & -1.12 & -0.40 & 0.29 \\
throttle (\%) & $40 \%$ & -0.35 & -1.12 & -0.46 & 0.45 \\
& $45 \%$ & -0.42 & -0.98 & -0.44 & 0.48 \\
& $50 \%$ & -0.47 & -0.73 & -0.45 & 0.65 \\
& $55 \%$ & -0.10 & -0.13 & -0.064 & 0.095 \\
& $60 \%$ & -0.15 & -0.14 & -0.26 & 0.37 \\
& $65 \%$ & -0.051 & -0.12 & -0.37 & 0.53
\end{tabular}

Table 14: Ribcraft USV Nomoto (23) parameter values (using linear fit of steady-state gains).

\begin{tabular}{cc|cc}
\multicolumn{2}{c}{ parameters } & $a_{22}$ & $b_{2}$ \\
\hline \hline & $20 \%$ & -0.23 & 0.12 \\
& $30 \%$ & -0.20 & 0.11 \\
$35 \%$ & -0.18 & 0.14 \\
$40 \%$ & -0.23 & 0.23 \\
throttle (\%) & $45 \%$ & -0.17 & 0.18 \\
$50 \%$ & -0.21 & 0.30 \\
$55 \%$ & -0.19 & 0.29 \\
$60 \%$ & -0.21 & 0.30 \\
$65 \%$ & -0.28 & 0.39
\end{tabular}

Table 15: Ribcraft USV speed (19) parameter values (using linear fit of steady-state gains).

\begin{tabular}{cc||cc} 
parameters & & $a$ & $b$ \\
\hline \hline & 1.0 & -0.026 & 0.0037 \\
& 1.5 & -0.095 & 0.021 \\
& 2.0 & -0.62 & 0.14 \\
Speed Region $(\mathrm{m} / \mathrm{s})$ & 2.5 & -0.28 & 0.043 \\
& 3.0 & -0.79 & 0.13 \\
& 3.5 & -0.17 & 0.033 \\
& 4.0 & -0.011 & 0.0031
\end{tabular}




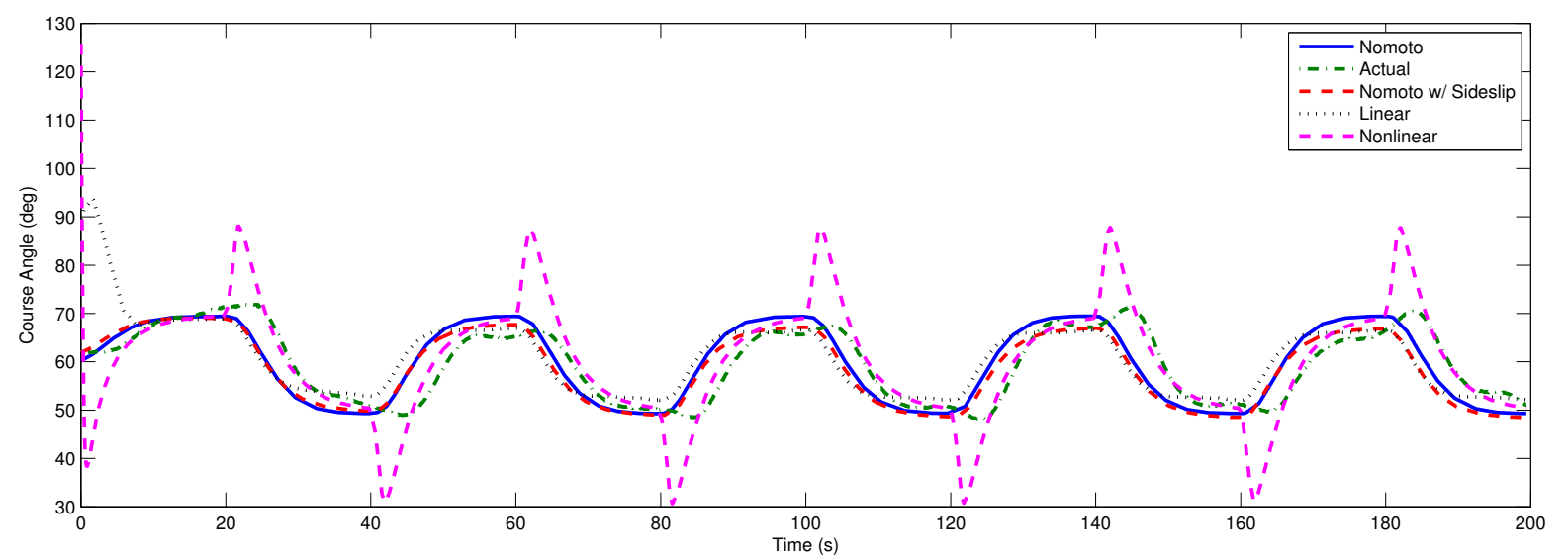

Figure 14: Time histories for course angle (actual and simulated) for the Ribcraft USV at $20 \%$ throttle.

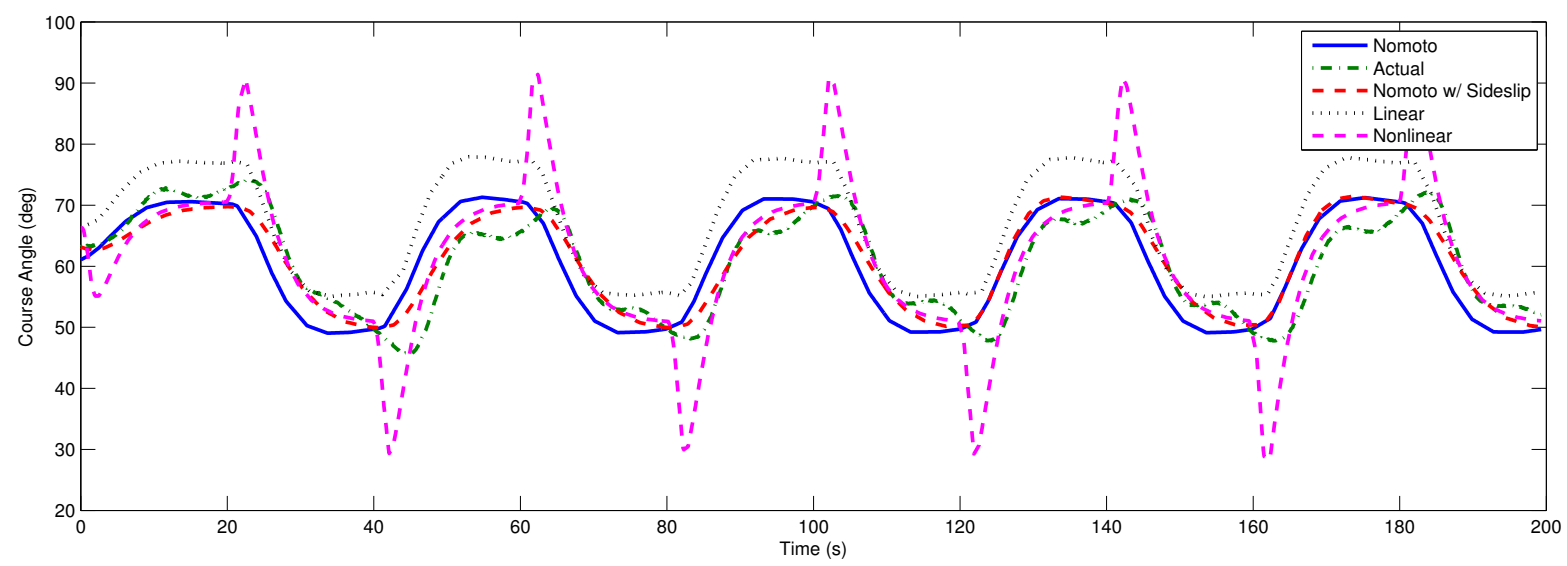

Figure 15: Time histories for course angle (actual and simulated) for the Ribcraft USV at $30 \%$ throttle. 


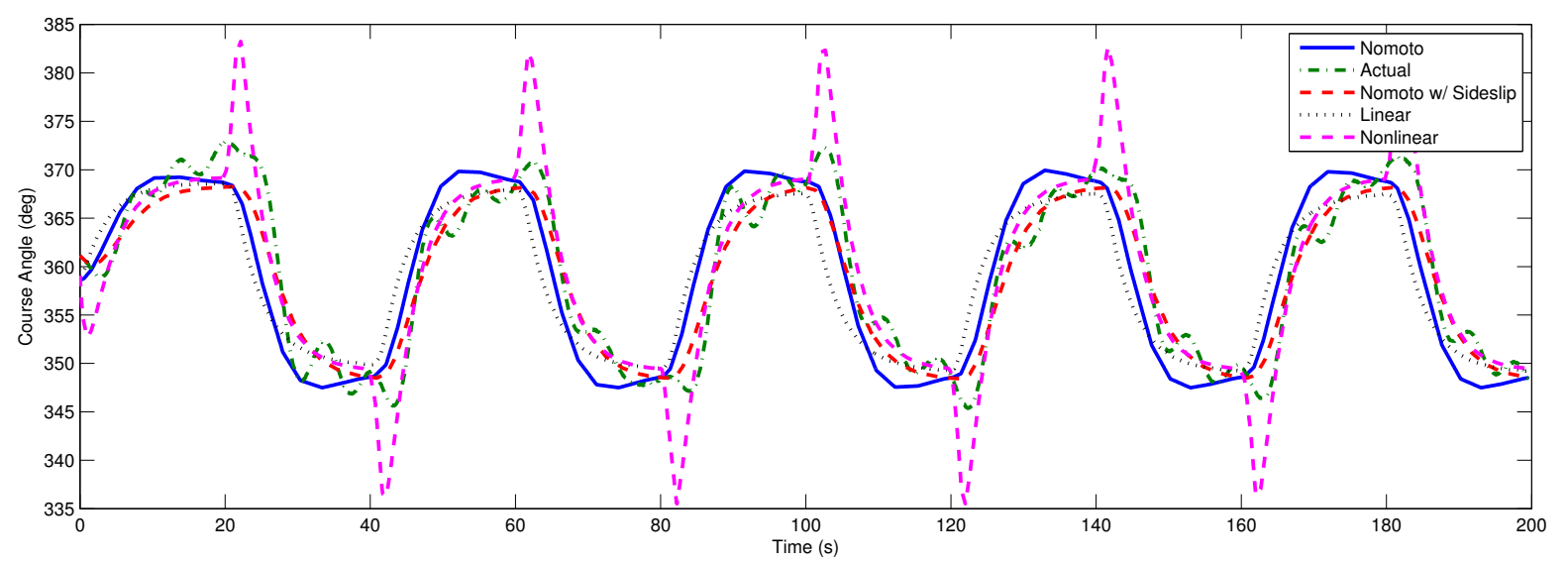

Figure 16: Time histories for course angle (actual and simulated) for the Ribcraft USV at $35 \%$ throttle.

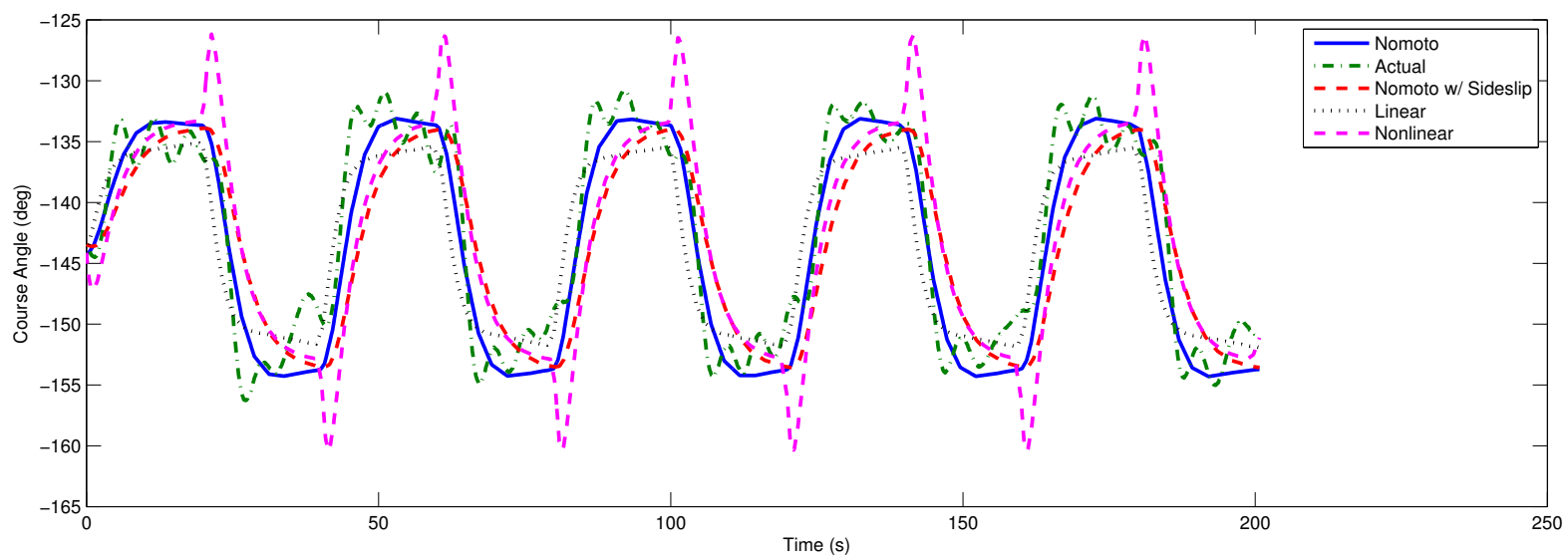

Figure 17: Time histories for course angle (actual and simulated) for the Ribcraft USV at $40 \%$ throttle. 


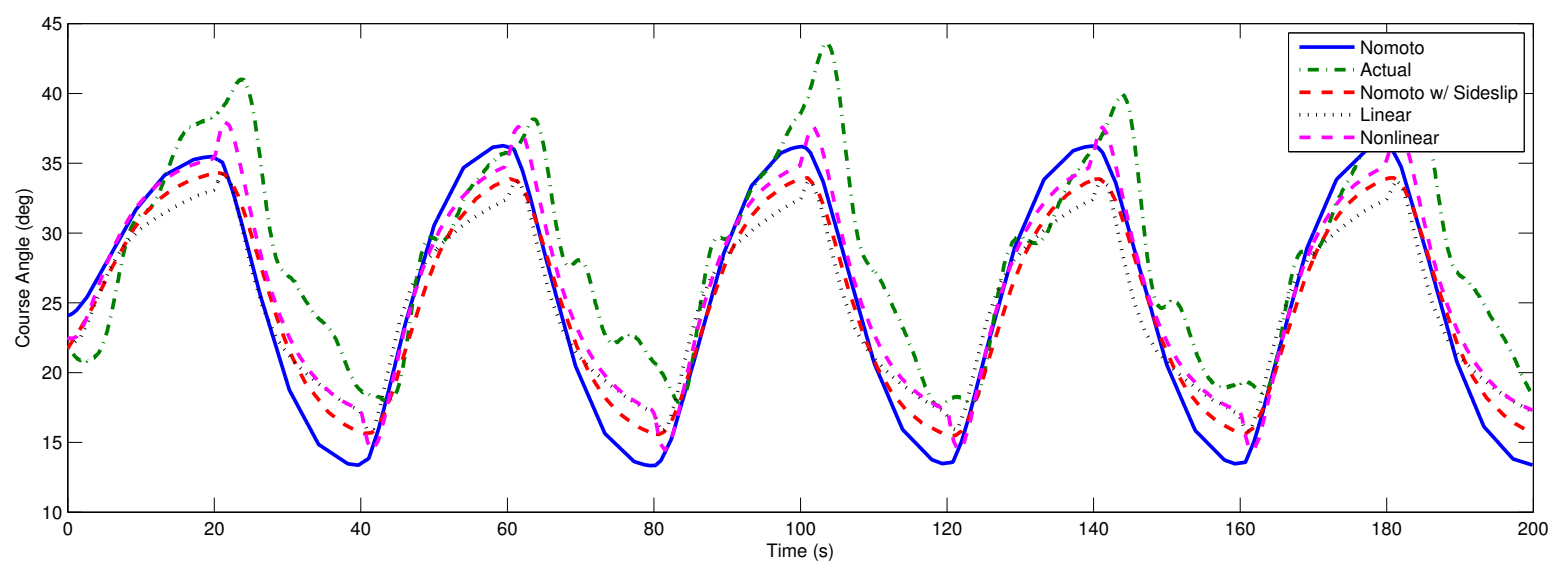

Figure 18: Time histories for course angle (actual and simulated) for the Ribcraft USV at $45 \%$ throttle.

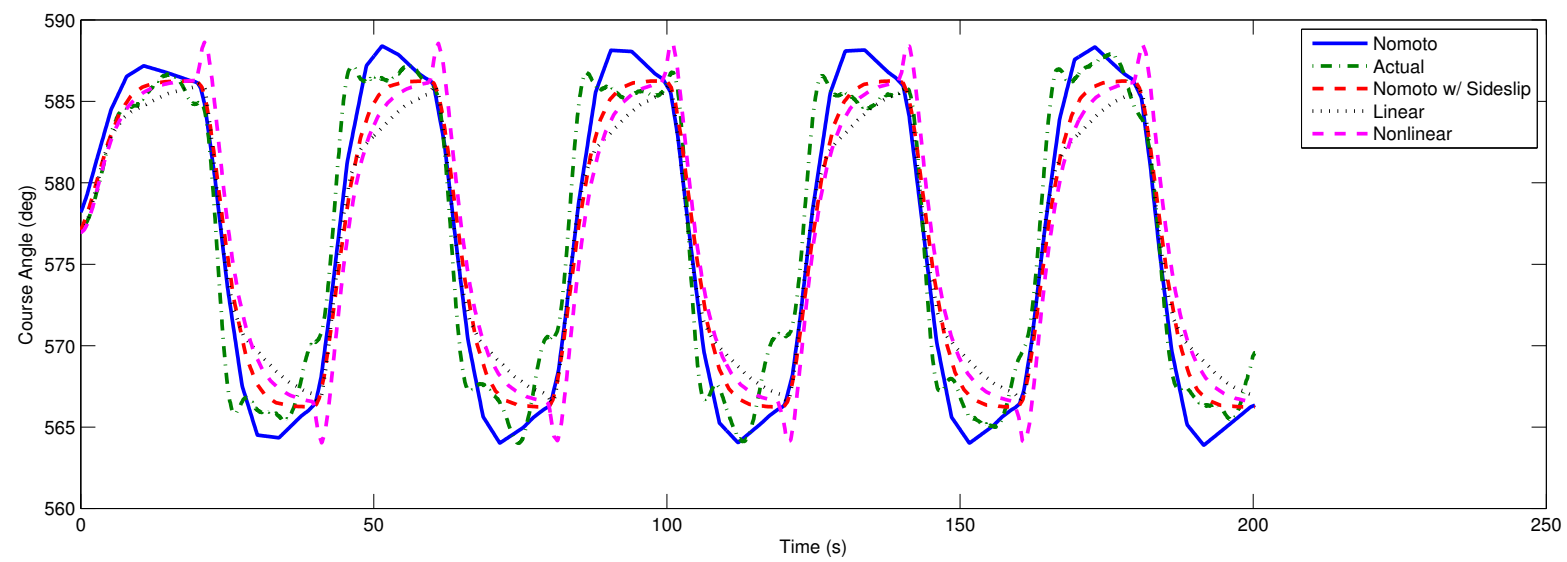

Figure 19: Time histories for course angle (actual and simulated) for the Ribcraft USV at $50 \%$ throttle. 


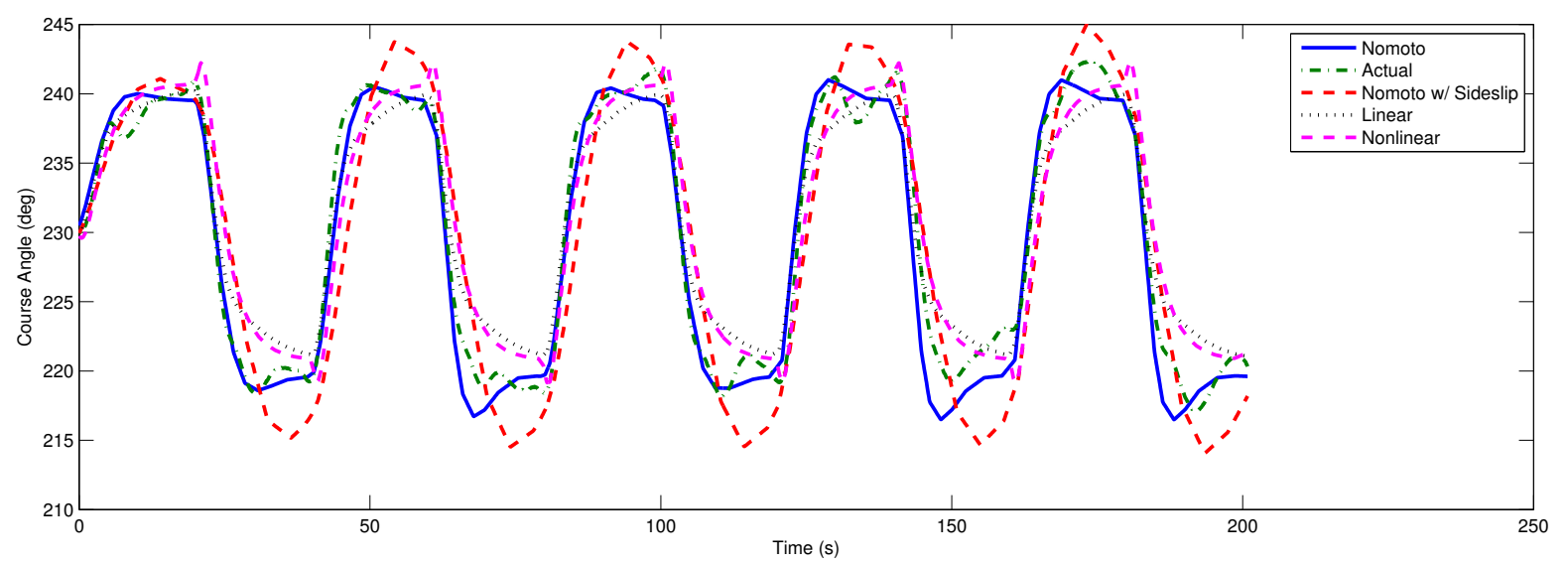

Figure 20: Time histories for course angle (actual and simulated) for the Ribcraft USV at $55 \%$ throttle.

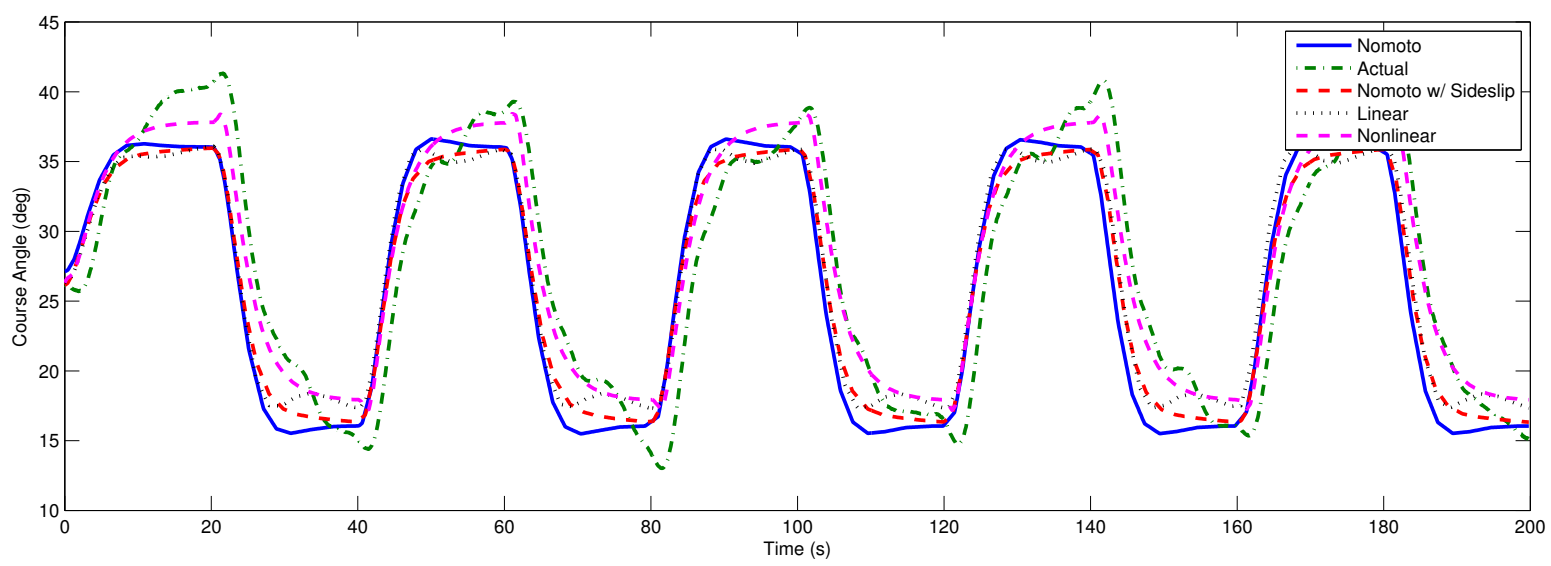

Figure 21: Time histories for course angle (actual and simulated) for the Ribcraft USV at $60 \%$ throttle. 
C. Sonnenburg, A. Gadre, D. Horner, S. Krageland, A. Marcus, D. J. Stilwell, and C. A. Woolsey

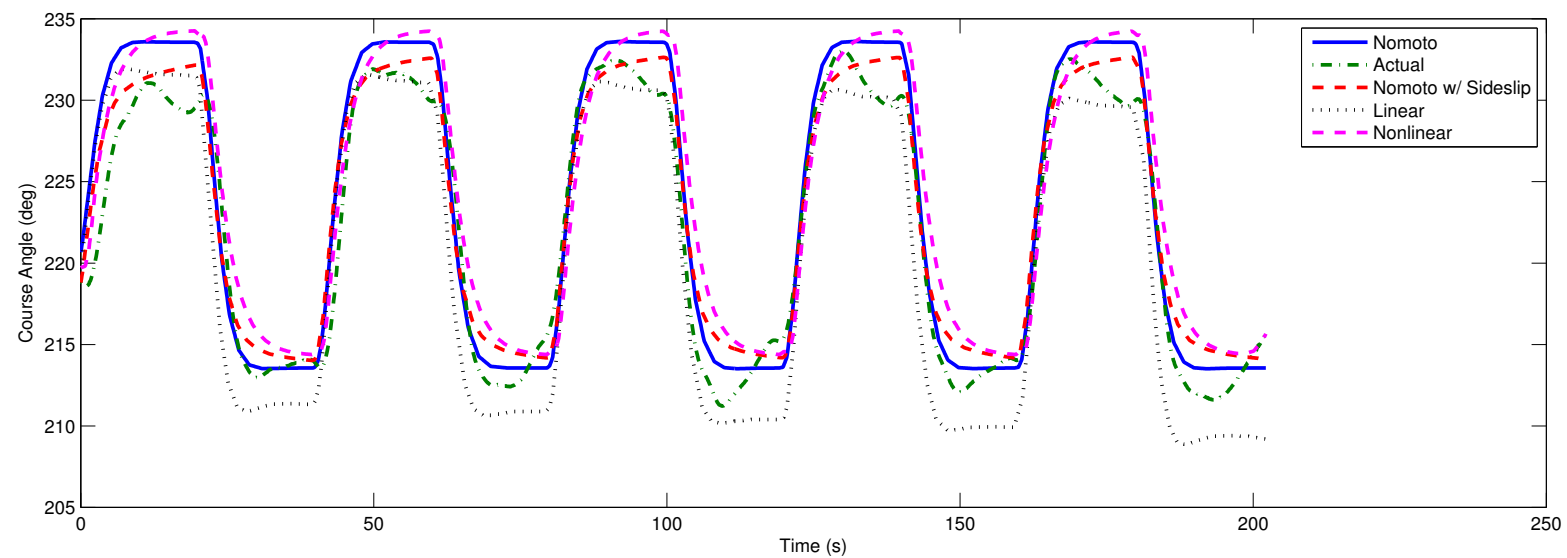

Figure 22: Time histories for course angle (actual and simulated) for the Ribcraft USV at $65 \%$ throttle. 


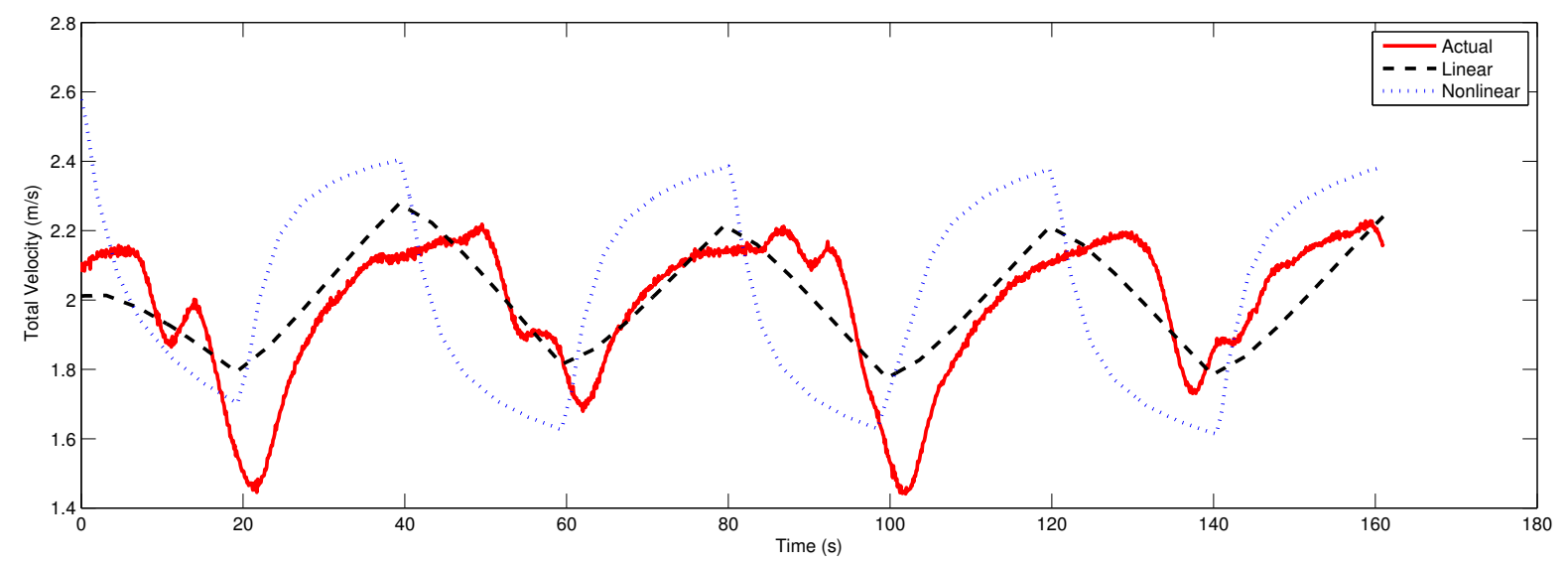

Figure 23: Time histories for speed (actual and simulated) for the Ribcraft USV for straight line travel around $1 \mathrm{~m} / \mathrm{s}$.

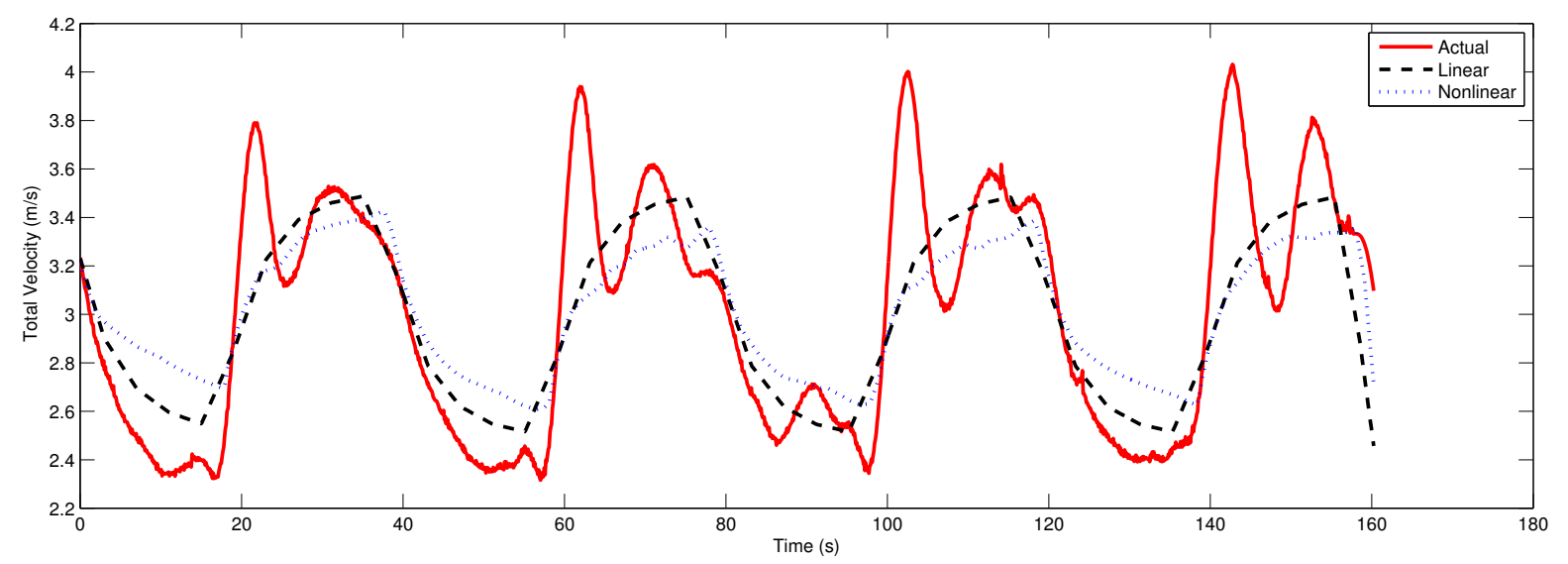

Figure 24: Time histories for speed (actual and simulated) for the Ribcraft USV for straight line travel around $1.5 \mathrm{~m} / \mathrm{s}$. 


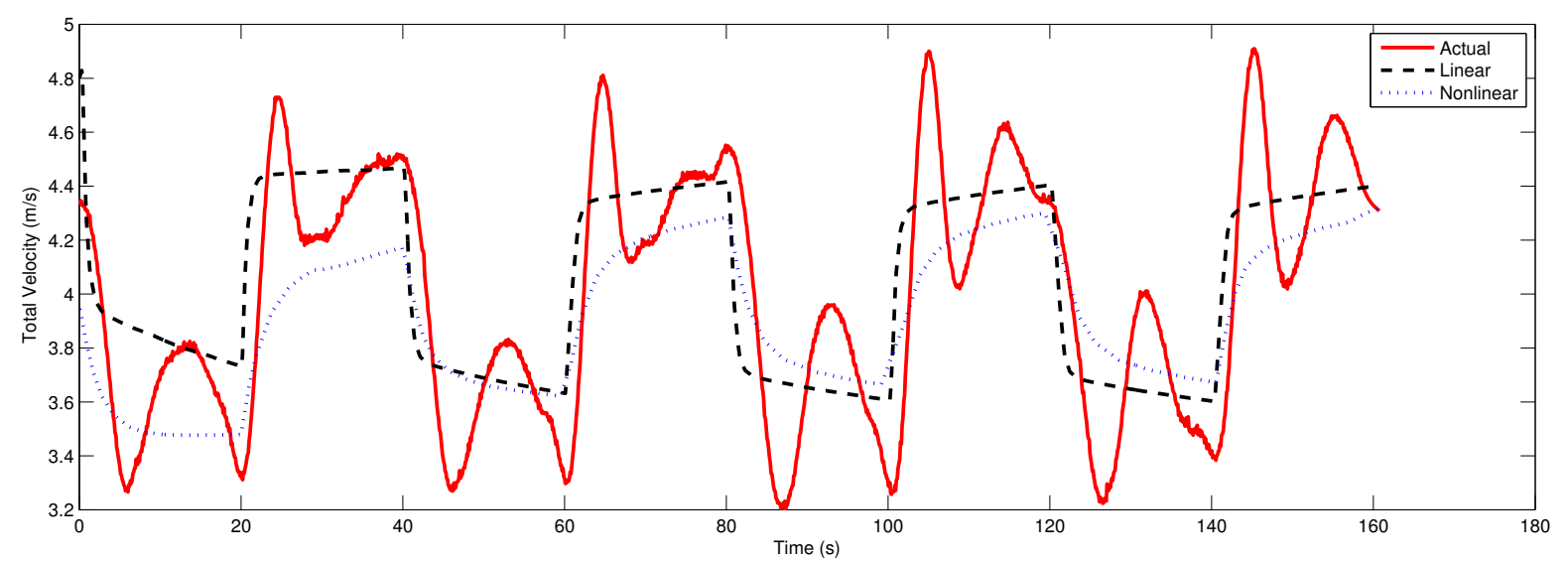

Figure 25: Time histories for speed (actual and simulated) for the Ribcraft USV for straight line travel around $2 \mathrm{~m} / \mathrm{s}$.

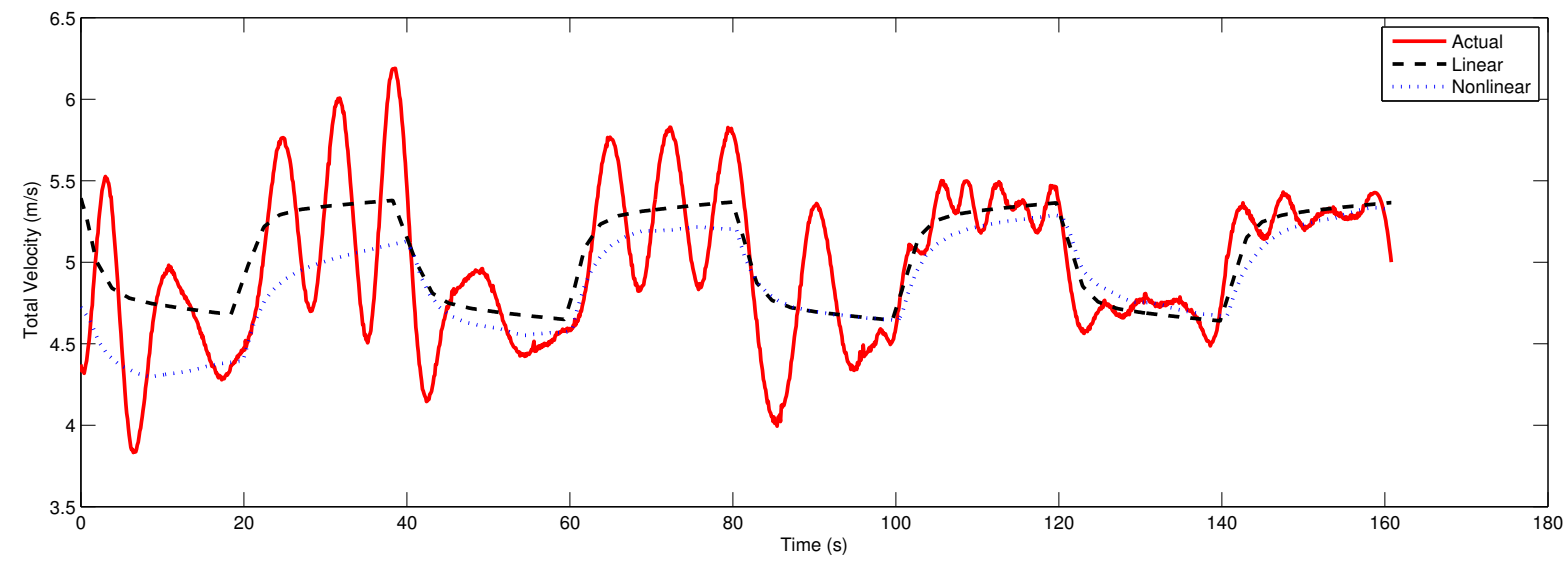

Figure 26: Time histories for speed (actual and simulated) for the Ribcraft USV for straight line travel around $2.5 \mathrm{~m} / \mathrm{s}$. 


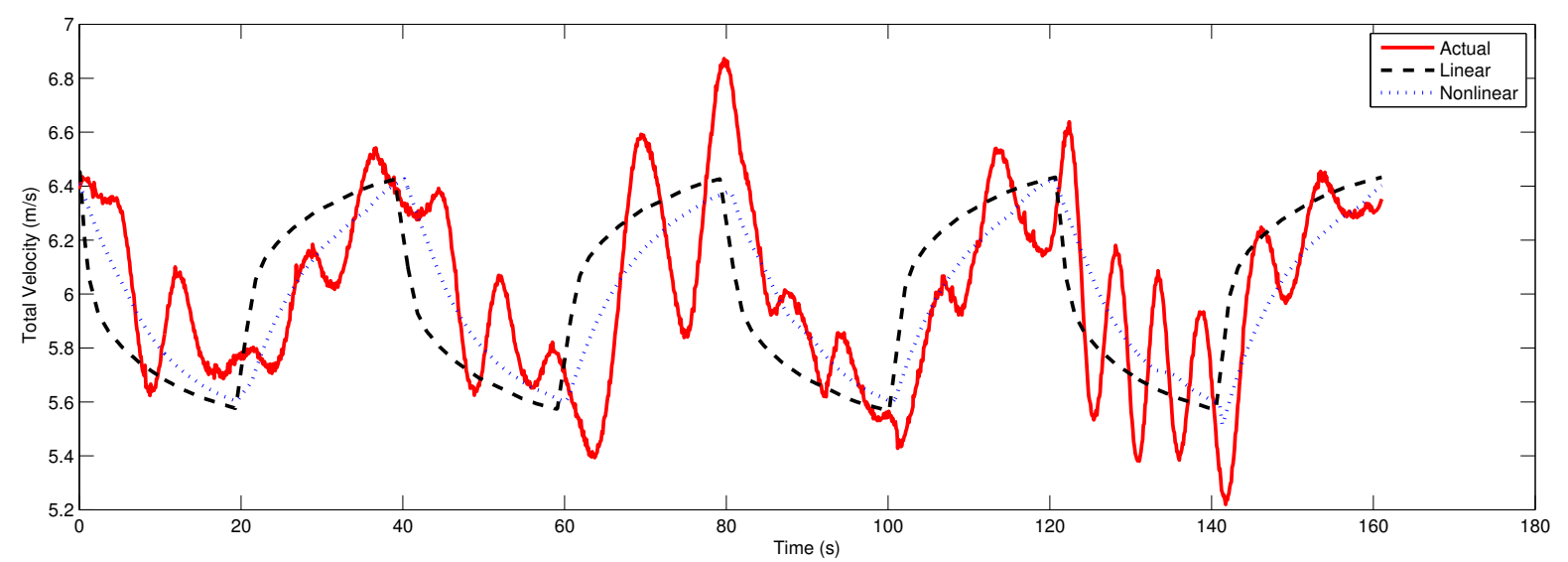

Figure 27: Time histories for speed (actual and simulated) for the Ribcraft USV for straight line travel around $3 \mathrm{~m} / \mathrm{s}$.

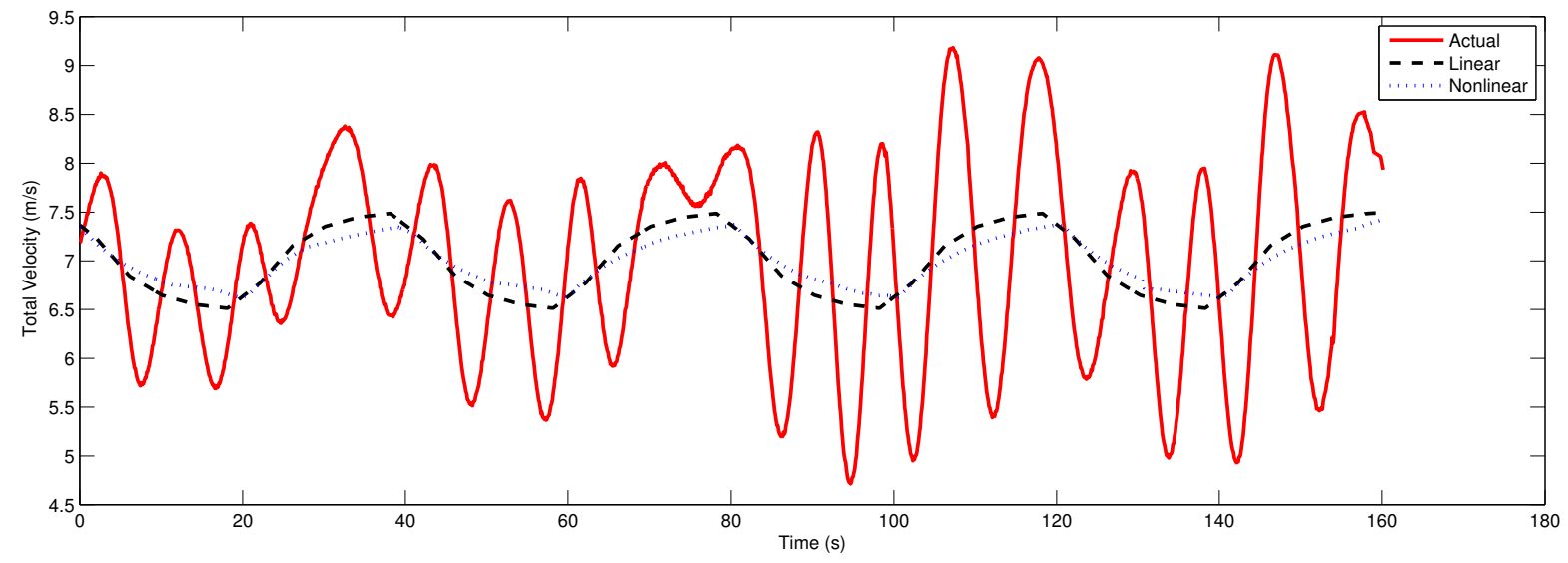

Figure 28: Time histories for speed (actual and simulated) for the Ribcraft USV for straight line travel around $3.5 \mathrm{~m} / \mathrm{s}$. 
C. Sonnenburg, A. Gadre, D. Horner, S. Krageland, A. Marcus, D. J. Stilwell, and C. A. Woolsey

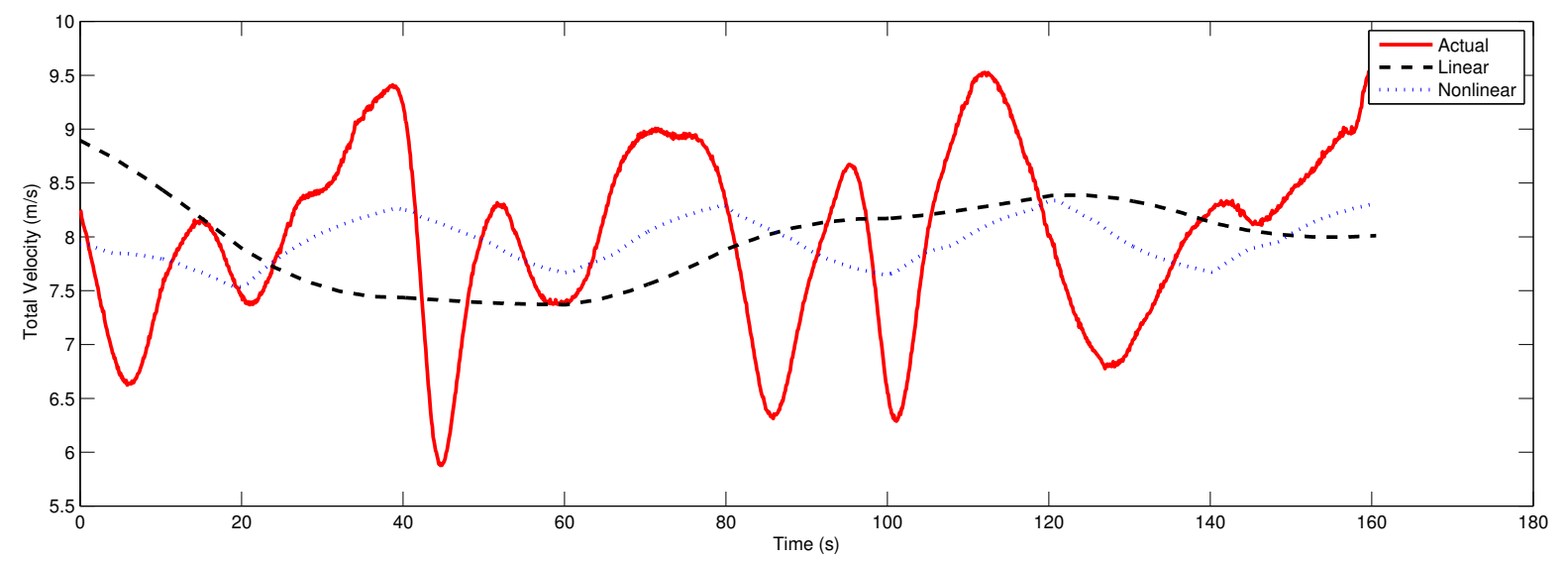

Figure 29: Time histories for speed (actual and simulated) for the Ribcraft USV for straight line travel around $4 \mathrm{~m} / \mathrm{s}$. 


\section{B Seafox USV Parameters and Time Histories}

Note that $a_{*}$ and $b_{*}$ refer to the elements $*$ in the state matrix $\boldsymbol{A}$ and input matrix $\boldsymbol{B}$ respectively. If $a$ and $b$ appear without a subscript, then the state and input matrices are scalars.

\section{B.1 Parameters}

Table 16: Seafox USV linear (21) parameter values (using linear fit of steady-state gains).

\begin{tabular}{cc||cccccc}
\multicolumn{2}{c}{ parameters } & $a_{11}$ & $a_{12}$ & $a_{21}$ & $a_{22}$ & $b_{1}$ & $b_{2}$ \\
\hline \hline \multirow{3}{*}{ throttle (\%) } & $15 \%$ & -0.011 & 0.00014 & 0.40 & -0.044 & -0.00023 & 0.027 \\
& $20 \%$ & -0.0028 & -0.00069 & 0.65 & -0.059 & 0.00029 & 0.036 \\
& $25 \%$ & 0.0019 & -0.00030 & 0.59 & -0.058 & 0.00019 & 0.039
\end{tabular}

Table 17: Seafox USV Nomoto-with-sideslip (25) parameter values (using linear fit of steadystate gains).

\begin{tabular}{cc||cccc}
\multicolumn{2}{c}{ parameters } & $a_{11}$ & $a_{13}$ & $a_{33}$ & $b_{3}$ \\
\hline \hline \multirow{3}{*}{ throttle $(\%)$} & $15 \%$ & -0.0029 & -0.015 & -0.059 & 0.028 \\
& $20 \%$ & -0.0000094 & -0.000053 & -0.071 & 0.033 \\
& $25 \%$ & -0.000000015 & -0.000000074 & -0.071 & 0.039
\end{tabular}

Table 18: Seafox USV Nomoto (23) parameter values (using linear fit of steady-state gains).

\begin{tabular}{cc||cc}
\multicolumn{2}{c}{ parameters } & $a_{22}$ & $b_{2}$ \\
\hline \hline & $15 \%$ & -0.059 & 0.028 \\
throttle $(\%)$ & $20 \%$ & -0.064 & 0.030 \\
& $25 \%$ & -0.037 & 0.020
\end{tabular}

\section{B.2 Time Histories}




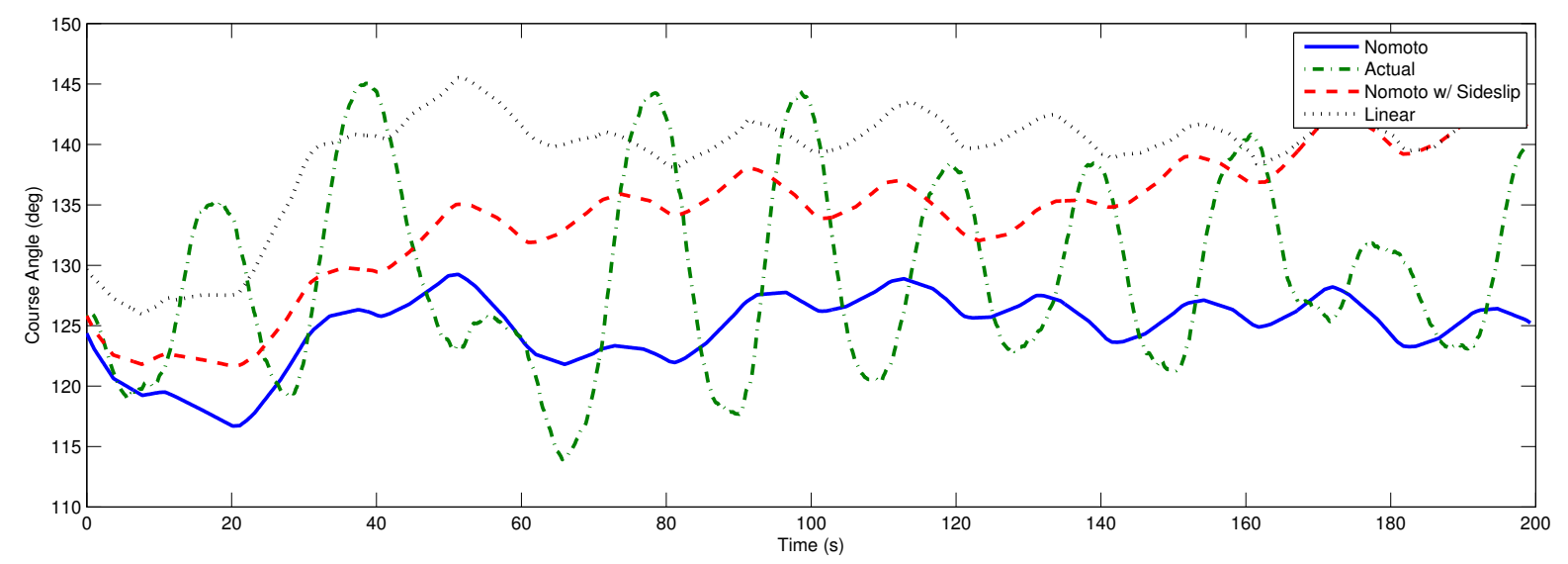

Figure 30: Time histories for course angle (actual and simulated) for the Seafox USV at 15\% throttle.

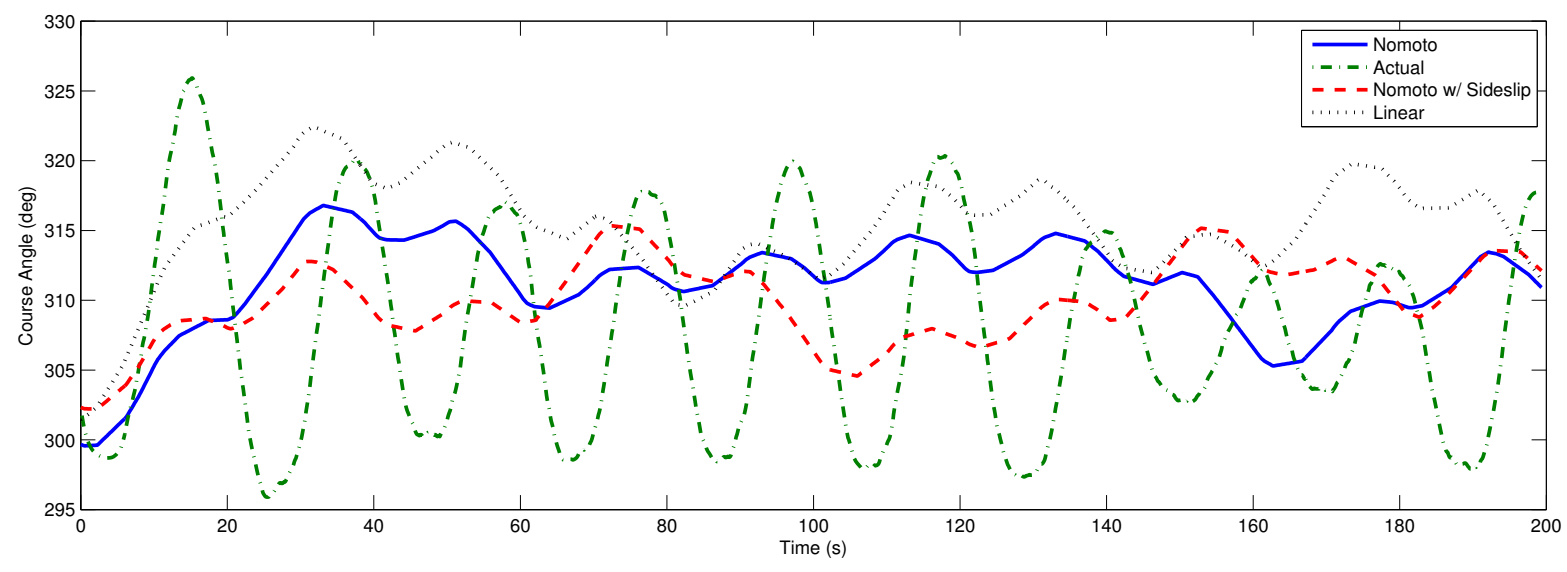

Figure 31: Time histories for course angle (actual and simulated) for the Seafox USV at $20 \%$ throttle. 
C. Sonnenburg, A. Gadre, D. Horner, S. Krageland, A. Marcus, D. J. Stilwell, and C. A. Woolsey

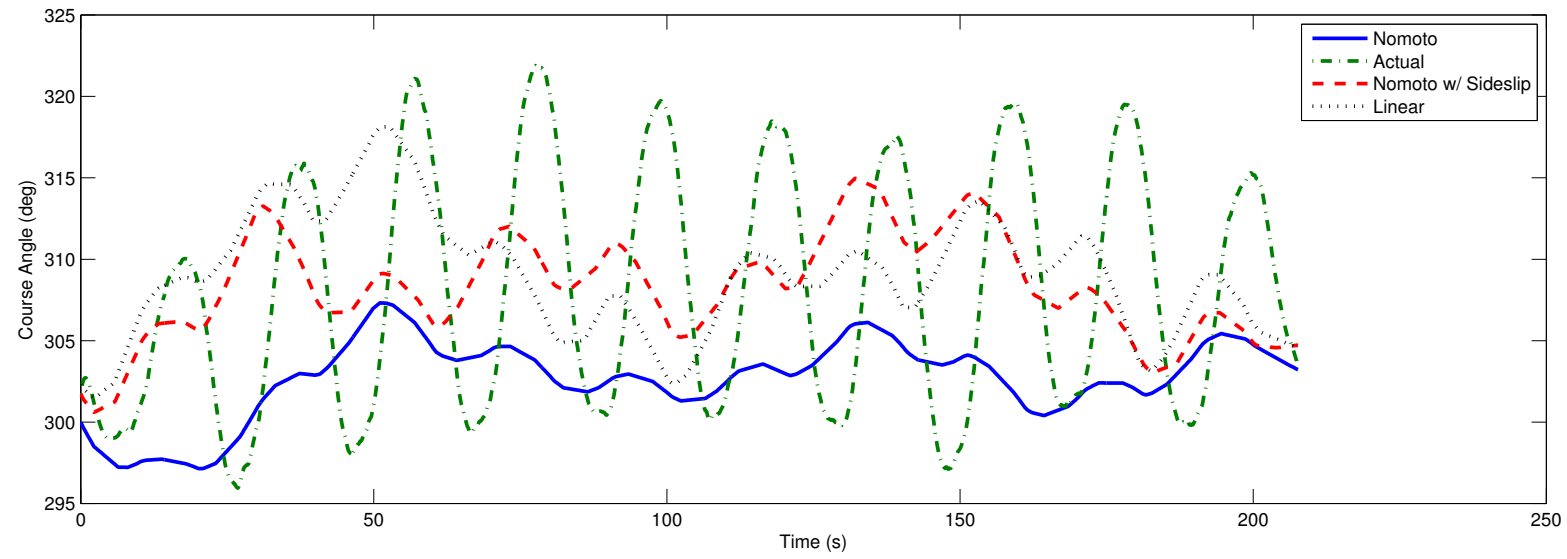

Figure 32: Time histories for course angle (actual and simulated) for the Seafox USV at 25\% throttle. 


\section{Pontoon USV Parameters and Time Histories}

Note that $a_{*}$ and $b_{*}$ refer to the elements $*$ in the state matrix $\boldsymbol{A}$ and input matrix $\boldsymbol{B}$ respectively. If $a$ and $b$ appear without a subscript, then the state and input matrices are scalars.

\section{C.1 Parameters}

Table 19: Pontoon USV linear (21) parameter values (using linear fit of steady-state gains).

\begin{tabular}{cc||cccccc}
\multicolumn{2}{c}{ parameters } & $a_{11}$ & $a_{12}$ & $a_{21}$ & $a_{22}$ & $b_{1}$ & $b_{2}$ \\
\hline \hline \multirow{2}{*}{ throttle (\%) } & $40 \%$ & -0.22 & -0.0044 & 12.41 & -0.19 & 0.0017 & 0.18 \\
& $50 \%$ & -0.041 & -0.0070 & 2.11 & -0.28 & 0.0046 & 0.20
\end{tabular}

Table 20: Pontoon USV Nomoto-with-sideslip (25) parameter values (using linear fit of steady-state gains).

\begin{tabular}{cc||cccc}
\multicolumn{2}{c}{ parameters } & $a_{11}$ & $a_{13}$ & $a_{33}$ & $b_{3}$ \\
\hline \hline \multirow{2}{*}{ throttle $(\%)$} & $40 \%$ & -0.50 & -1.098 & -0.56 & 0.35 \\
& $50 \%$ & -0.59 & -1.18 & -0.29 & 0.20
\end{tabular}

Table 21: Pontoon USV Nomoto (23) parameter values (using linear fit of steady-state gains).

\begin{tabular}{cc||cc}
\multicolumn{2}{c}{ parameters } & \multicolumn{1}{c}{$a_{22}$} & $b_{2}$ \\
\hline \hline \multirow{2}{*}{ throttle $(\%)$} & $40 \%$ & -0.53 & 0.33 \\
& $50 \%$ & -0.72 & 0.49
\end{tabular}

\section{C.2 Time Histories}




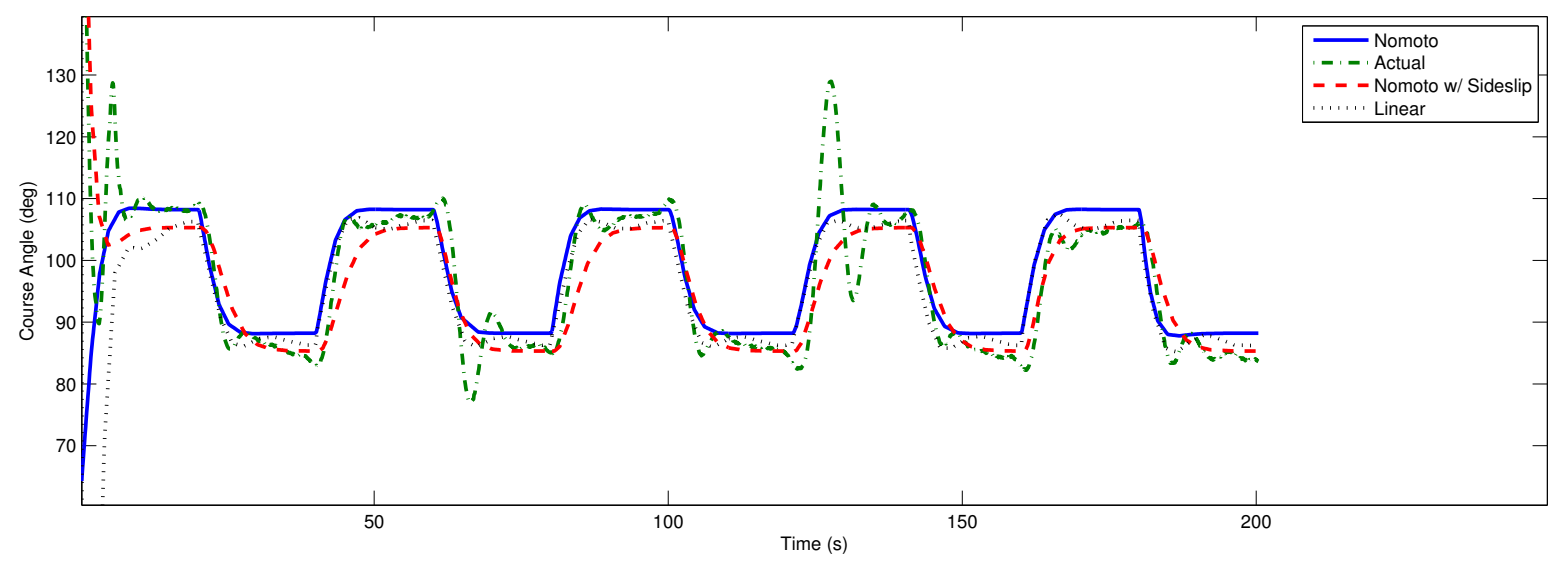

Figure 33: Time histories for course angle (actual and simulated) for the Pontoon USV at $40 \%$ throttle.

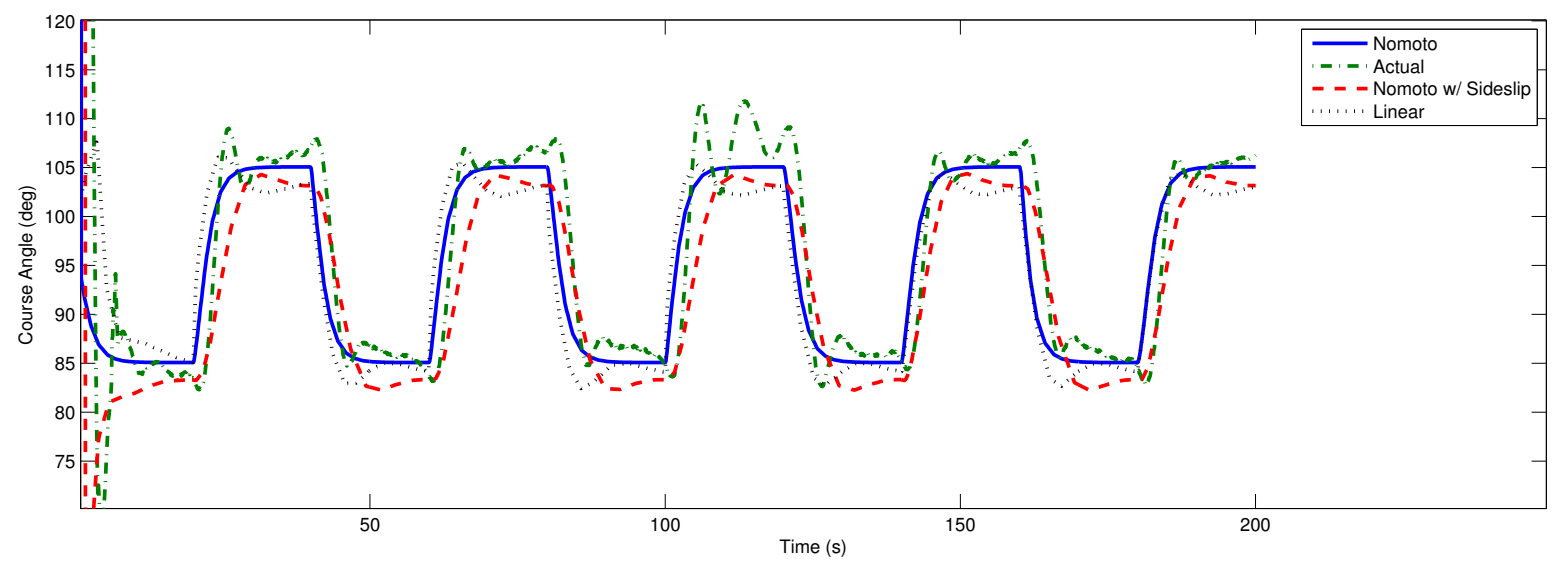

Figure 34: Time histories for course angle (actual and simulated) for the Pontoon USV at $50 \%$ throttle. 